\title{
Phytosociological analysis of beech forests in the Žumberak and Samobor highlands (Croatia)
}

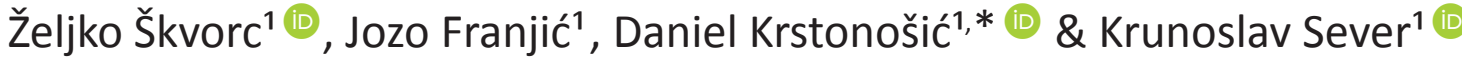

Key words: Vegetation, Relevés, Environmental gradients, Fagus sylvatica, Forest communities, Croatia.

Ključne besede: vegetacija, popisi, okoljski gradient, Fagus sylvatica, gozdne združbe, Hrvaška.
Received: 08. 02. 2020

Revision received: 24. 03. 2020

Accepted: 25. 03. 2020

\begin{abstract}
The Žumberak and Samobor highlands are situated in the north-western part of Croatia where the characteristics of the Dinarides, the Alps and the Pannonian Plain meet. The greater part of the area is occupied by beech forests. The aim of the study was to determine the syntaxonomic affiliation of these forest communities, and to explore their floristic and main ecological features. Numerical analyses of floristic compositions were conducted on a data-set consisting of 144 new relevés and 99 relevés from the existing literature. Relevés were made following the standard Braun-Blanquet method. For descriptions of ecological conditions Ellenberg's indicator values were used. Six plant associations and two subassociations of beech forests were established in Luzulo-Fagion and Aremonio-Fagion alliances. The higher altitudes of the studied area are occupied by ass. Cardamini savensi-Fagetum, whereas the lower altitudes are occupied by ass. Lamio orvalae-Fagetum and Hacquetio-Fagetum. Ass. Hacquetio-Fagetum spreads on southern slopes and ridges, whereas ass. Lamio orvalae-Fagetum occurs in ditches and on northern slopes. In the warmer habitats with shallow soils on a dolomite base ass. Ostryo-Fagetum occurred. A small part of the area is characterized by silicate substrate which is occupied by ass. Luzulo-Fagetum and Gentiano asclepiadeae-Fagetum.
\end{abstract}

\section{Izvleček}

Žumberak-Samoborsko gorje se nahaja v severozahodnem delu Hrvaške, kjer se združi vpliv Dinarskega gorstva, Alp in Panonske nižine. Večji del območja poraščajo bukovi gozdovi. Namen raziskave je bil sintaksonomsko opredeliti gozdne združbe in proučiti njihovo vrstno sestavo in glavne ekološke značilnosti. Numerične analize floristične sestave smo naredili s podatki iz 144 novih popisov in $99 \mathrm{iz} \mathrm{literature.} \mathrm{Popise} \mathrm{smo} \mathrm{naredili} \mathrm{v} \mathrm{skladu} \mathrm{s} \mathrm{standardno} \mathrm{Braun-Blanquetovo}$ metodo. Za opis ekoloških razmer smo uporabili Ellenbergove indikatorske vrednosti. Ugotovili smo šest asociacij in dve subasociaciji bukovih gozdov iz zvez Luzulo-Fagion in Aremonio-Fagion. Na višjih nadmorskih višinah uspeva ass. Cardamini savensi-Fagetum, na nižjih pa Lamio orvalae-Fagetum in HacquetioFagetum. Asociacija Hacquetio-Fagetum se pojavlja na južnih pobočjih in grebenih, medtem ko asociacijo Lamio orvalae-Fagetum najdemo v jarkih in na severnih pobočjih. Na toplejših rastiščih na plitkih tleh in dolomitni podlagi uspevajo sestoji asociacije Ostryo-Fagetum. Manjši del območja je na silikatni podlagi, kjer najdemo sestoje asociacij Luzulo-Fagetum in Gentiano asclepiadeae-Fagetum. 


\section{Introduction}

The Žumberak and Samobor highlands are situated in the western part of Croatia and together with the Gorjanci range in Slovenia comprise a hilly massif on the edge of the Pannonian plain, between the south-eastern Alps and north-western Dinaric Mountains (Figure 1). Phytogeographically, it is a transitional area with Illyric, West-European, Balkan-Apennine and Pannonian plant species, and rich with diverse flora and vegetation (Horvat 1929, Trinajstić 1995). Because of its great biological and geomorphological diversity and its unspoilt nature most of the Croatian part of the massif has been declared a Nature Park. The whole area today is covered mostly by forest vegetation, currently comprising almost $80 \%$ and gradually increasing (Vrbek 2010, Jelaska et al. 2005). During the last decades the entire area has been strongly affected by depopulation which has resulted in the abandonment of agricultural land as well as a decrease of the anthropogenic impacts on forests. Because of that a large part of the area is affected by secondary succession.

There is a long tradition in botanical and vegetation research of the Samobor area (Šugar 1972, Trinajstić 1995), while the area of Žumberak is much less researched (Vrbek 2005). Since almost $60 \%$ of the area is covered by beech forest (Jelaska et al. 2005) there is a strong need for systematic evaluation and ecological

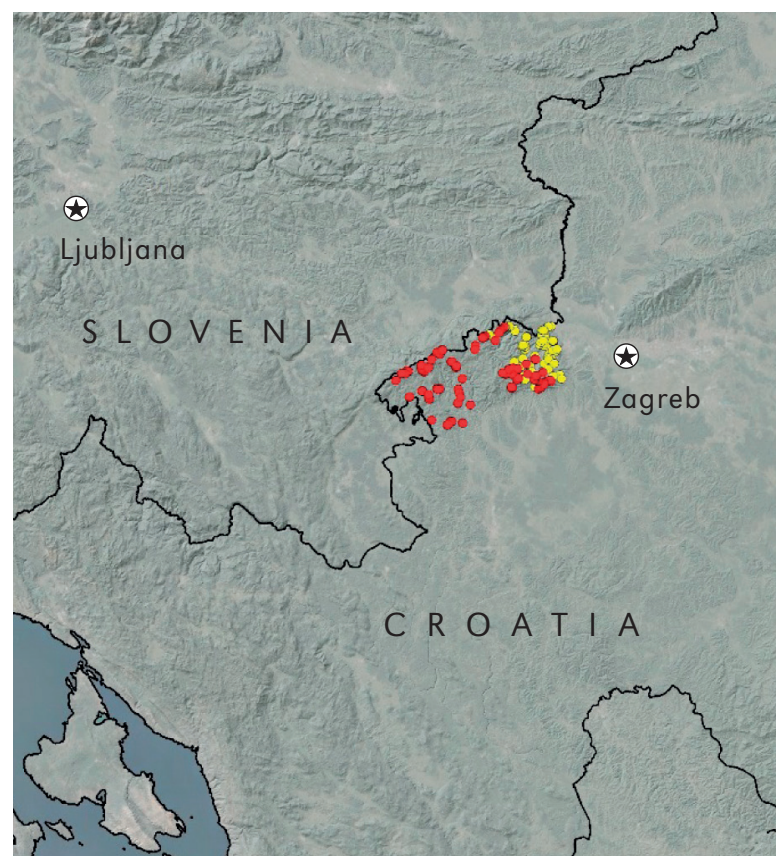

Figure 1: The study area with relevés locations. Red - new relevés, yellow - literature relevés.

Slika 1: Raziskovano območje z lokacijami popisov. Rdeče - novi popisi, rumeno - popisi iz literature. characterization of the beech forest vegetation. There is a considerable amount of data in the literature on the forest vegetation of the Samobor mountain belt (Horvat 1938, Šugar 1972, Pavletić et al. 1982, Vukelić et al. 2003, Trinajstić 2004, Trinajstić \& Cerovečki 2005). On the other hand, the literature on forest vegetation of Žumberak is very poor. However, forest vegetation in the neighbouring Slovenian area (Gorjanci) has been explored and elaborated in much more detail (Košir 1979, Marinček 1987, Marinček et al. 2001).

The aim of this study was to determine the syntaxonomic affiliation of beech forest communities of the Žumberak and Samobor highlands, and to explore their floristic and main ecological features.

\section{Methods}

\section{Study area}

The study area is comprising Žumberak and Samobor highlands (Croatia) which together with Gorjanci Mt. (Slovenia) makes $40 \mathrm{~km}$ long and $30 \mathrm{~km}$ wide dissected massif between the rivers Sava (on the north), Kupa (on the east), and Krka (on the west), with an elevation range between $180 \mathrm{~m}$ (Kupa River valley) and $1178 \mathrm{~m}$ a. s. l. at the top of St. Gera.

The largest part of the area is built of karstified carbonate beds (limestones, dolomites and breccias) of Triassic, Jurassic and Cretaceous age, with numerous karst relief forms, such us dolines, blind valleys, caves and pits (Buzjak 2002). In lesser amount are present clay shales, quartz conglomerates and sandstones of Paleozoic age (Vukelić et al. 2003). Structurally, whole area is in a border zone between the Inner Dinarides and the Zagorje-MidTransdanubian shear zone, overthrusting on the External Dinarides (Pamić \& Tomljenović 1998).

Climate of the area is temperate continental. The mean annual temperature varies between $6{ }^{\circ} \mathrm{C}$ in the highest mountain parts and $11{ }^{\circ} \mathrm{C}$ in the lowest southeastern parts (Zaninović et al. 2004). The mean air temperature of January varies from $-1{ }^{\circ} \mathrm{C}$ to less than $-2{ }^{\circ} \mathrm{C}$, and the mean air temperature in July varies from $20^{\circ} \mathrm{C}$ to less than $18^{\circ} \mathrm{C}$. The highest precipitation occurs in June and October, and the lowest in March with an average annual precipitation between 1100 and $1700 \mathrm{~mm}$ (Penzar \& Penzar 1982).

The study area is mostly covered by forest vegetation dominated by forests of sessile oak and common hornbeam (Erythronio-Carpinion) in colline vegetation belt and beech forests (Aremonio-Fagion) in submontane and montane vegetation belt. However, due to the complex relief and geological structure some other acidophilous 
and termophilous forest communities occur extrazonally or azonally. Coniferous forests (Picea abies and Pinus sylvestris) were planted after World War II, but their actual total surface is less than $1 \%$. The total surface of dry grasslands (Bromion erecti), mesophilous grasslands (Arrhenatheretalia elatioris) and heathlands (Calluno-Genistion pilosae) is rapidly decreasing due to abandonment of traditional land use and their cover is now probably less than $12 \%$ of total land surface (Horvat 1962, Šugar 1972, Jelaska et al. 2005, Vrbek 2010).

\section{Vegetation and environmental data}

Vegetation was sampled and relevés were elaborated according to standard Central European method (BraunBlanquet 1964, Westhoff \& van der Maarel 1973). This study is based on a data-set consisting of 243 relevés. 99 relevés were used from the available literature (Horvat 1938, Šugar 1972, Pavletić et al. 1982, Trinajstić 2004, Trinajstić \& Cerovečki 2005, Vukelić et al. 2003), and 144 are new and were made in 2010. The area of new relevés is $400 \mathrm{~m}^{2}$, while the area of literature relevés vary between 100 and $1000 \mathrm{~m}^{2}$ (average $299 \mathrm{~m}^{2}$, mod and median $400 \mathrm{~m}^{2}$ ). The relevés were stored in TURBOVEG (Hennekens \& Schaminée 2001). Taxonomic nomenclature follows Nikolić et al. (2020). Nomenclatural decisions follow the fourth edition of the International Code of Phytosociological Nomenclature (Theurillat et al. 2020). The names of higher syntaxa follow Škvorc et al. (2017).

Ellenberg's indicator values (EIV) were used for ecological interpretation of vegetation patterns (Ellenberg et al. 1992). Unweighted mean indicator values were calculated for each relevé using JUICE software (Tichý 2002). Aspects were transformed according to Beers et al. (1966).

\section{Data analysis}

The numerical classification of the relevés, based on their species composition was performed in the PC-ORD (McCune \& Mefford 2006) using cluster analysis (Beta flexible $/ \beta=-0.25 /$ for group linkage with Sørensen index as the distance measure). The OptimClass method for identifying the optimal partition was used (Tichý et al. 2010).

Diagnostic species of obtained communities were determined by calculating fidelity using the phi $(\Phi)$ coefficient. Only species with $\Phi>0.3$ and a probability under random expectation of the observed pattern of species occurrence lower than 0.001 (Fisher's exact test) were considered diagnostic. To calculate fidelity, the number of relevés for each order or alliance was virtually standardized to equal size (Tichy \& Chytry 2006).
To check the communities' differentiation and to explore relationship with environmental variables NonMetric Multidimensional Scaling (NMDS) was applied using the Bray-Curtis dissimilarity matrix on square-root transformed percentage cover values. NMDS with passive projection of environmental variables was performed using the R package 'vegan' (https://cran.r-project.org/ web/packages/vegan) operated through the JUICE software (Tichý 2002). Plot scores on the first two NMDS axes correlated with environmental variables. Correlations for Ellenberg indicator values were corrected using the modified permutation test (Zelený \& Schaffers 2012). Box \& Whiskers diagrams of environmental variables were made using STATISTICA for WINDOWS 7.0 (Statsoft, Inc. 2005).

\section{Results and Discussion}

We recorded 293 taxa of vascular plants with Fagus sylvatica, Rubus hirtus, Galium odoratum, Hedera helix, Acer pseudoplatanus, Cyclamen purpurascens, Pulmonaria officinalis, Sanicula europaea, and Aposeris foetida being the most common ones (frequency $\geq 50 \%$ ). Numerical classification separated studied relevés of beech forests in nine clusters which are considered as seven associations and two subassociations in Luzulo-Fagion and Aremonio-Fagion alliances (Figure 2). According to the syntaxonomic system of higher units (Škvorc et al. 2017), these syntaxa can be classified as follows:

\section{Carpino-Fagetea sylvaticae Jakucs ex Passarge 1968}

Luzulo-Fagetalia sylvaticae Scamoni et Passarge 1959

Luzulo-Fagion sylvaticae Lohmeyer et Tx. in Tx. 1954 Luzulo luzuloidi-Fagetum Meusel 1937

Gentiano asclepiadeae-Fagetum sylvaticae ass. nova

Fagetalia sylvaticae Pawłowski 1928

Aremonio-Fagion (Horvat 1938) Borhidi in Török et al. 1989

Ostryo-Fagenion Borhidi 1963

Ostryo-Fagetum M. Wraber ex Trinajstić 1972

Epimedio-Fagenion Marinček et al. 1993

Vicio oroboidi-Fagetum Horvat (1938) Pócs et Borhidi in Borhidi 1960

Note: Horvat (1938) divided the association 'Fagetum silvaticae croaticum' into two geographical races ('boreale' and 'australe'). 'Boreale' is further divided into two syntaxa, as 'montanum' and 'abietosum', and 'montanum' is then further subdivided into 'lathyretosum' and 'corydaletosum'. The rank of these syntaxa is sometimes doubtful because Horvat gave them different ranks in different parts of the article. In the main text he consid- 
ered 'montanum' as a subassociation as well as 'group of subassociations' (p. 197), while he included 'lathyretosum' as a subassociation of 'montanum' (p. 199). In the summary chapter 'montanum' is considered as a subassociation and he states that this subassociation could be divided into several facies that are not syntaxonomically equivalent and could well be united into a group of facieses or even subassociations (e.g, Fagetum croaticum montanum lathyretosum, Fagetum croaticum montanum corydaletosum etc.) (p. 295).

Moreover, when he refers to the subassociation montanum he described the acidophilous facies with Luzula luzuloides that is separated from two 'sociological entities', as Fagetum croaticum montanum lathyretosum and Fagetum croaticum montanum corydaletosum that are differentiated by some sociologically important species and can probably be treated as subassociations with some facies (p. 199).

Hence we cannot accept the opinion of Willner (2002) and Theurillat et al. (2020) who treat these two subassociations as facieses. We considered both subassociations as validly published. Art. 3d applies here, stating that in the case of several subassociations that are hierarchically subordinated the lowest level is validly described [Art. 3d]. In the same publication, Horvat (1938) used the alternative name of Fagetum croaticum as Lamio orvalae-Fagetum [Art. 3j, 39] and therefore subassociations Lamio orvalae-Fagetum lathyretosum and Lamio orvalae-Fagetum corydaletosum are validly published. Lamio orvale-Fagetum lathyretosum serves as basionym (Def. XI) of Vicio oroboidi-Fagetum. Borhidi (1960) was of the same opinion and described the association Vicio oroboidi-Fagetum and also Marinček et al. (1993), who typified it, as well as many other authors (Borhidi 1963, Marinček \& Čarni 2002, Vukelić \& Baričević 2002, Trinajstić 2008, Vukelić 2012, Šilc \& Čarni 2013).

Hacquetio-Fagetum Ž. Košir ex Borhidi 1963 aceretosum obtusati subass. nova

Lamio orvalae-Fagenion Borhidi ex Marinček et al. 1993

Lamio orvalae-Fagetum Horvat 1938

Note: Marinček et al. (1993) agreed with Borhidi (1963) who corrected the illegitimate name Fagetum silvaticae croaticum boreale montanum described by Horvat (1938) [Art. 34a] to Lamio orvalae-Fagetum, which is later accepted in many literature sources (Marinček \& Čarni 2002, Vukelić \& Baričević 2002, Trinajstić 2008, Vukelić 2012, Šilc \& Čarni 2013). However, Horvat (1938) also proposed on p. 212 the name 'Fageto-Lamietum orvalae' as an alternative for the Fagetum silvaticae croaticum. So this is an alternative, validly published name [Art. 3j, Example 2; Art. 39a, Example 2], typified by Marinček et al. (1993).

fraxinetosum orni subass. nova

Cardamini savensi-Fagetum Ž. Košir ex Marinček et al. 1993

\section{Acidophilous communities}

A small part of the studied area is characterized by a silicate substrate where acidophilous beech communities occur. These communities in the studied area are classified in two clusters (Figure 2). The first is corresponding with ass. Luzulo-Fagetum with 14 relevés from the literature (Šugar 1972, Vukelić et al. 2003) as well as five new relevés (Table 3). The second cluster includes literature relevés of Blechno-Fagetum in Samobosko gorje area (Šugar 1972, Vukelić et al. 2003).

This association was invalidly described by Horvat (1950) under the name Fageto-Blechnetum [Art. 7]. However, Tüxen \& Oberdorfer (1958) validly published Blechno-Fagetum from Spain and used the epithet ibericum to differentiate from Horvat's syntaxa. Rivas-Martínez (1962) corrected this illegitimate name to Blechno-Fagetum [Art. 34a]. Furthermore, Marinček (1970) validated Horvat's name and produced a later homonym [Art. 31] (see also Trinajstić 2004).

Considering all the literature data, it is not correct to use the association name Blechno-Fagetum for acidophilous forest communities in SE and Central Europe (Marinček \& Zupančić 1995). Blechno-Fagetum sylvaticae (Tüxen et Oberdorfer 1958) Rivas-Martínez 1962 comprises acidophilous beech forests of the atlantic regions of

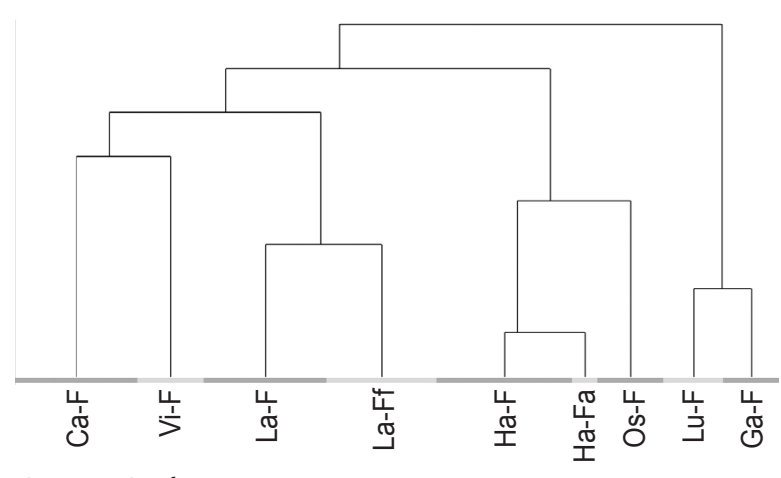

Ca-F Cardamini savensi-Fagetum

Vi-F Vicio oroboidi-Fagetum

La-F Lamio orvalae-Fagetum

La-Ff Lamio orvalae-Fagetum fraxinetosum orni

Ha-F Hacquetio-Fagetum

Ha-Fa Hacquetio-Fagetum aceretosum obtusati

Os-F Ostryo-Fagetum

Lu-F Luzulo luzuloidi-Fagetum

Ge-F Gentiano asclepiadeae-Fagetum

Figure 2: Dendrogram of beech forest communities of Žumberak and Samoborsko gorje area. Beta flexible $(\beta=-0.25)$ with Sørensen index as a distance measure.

Slika 2: Dendrogram bukovih gozdnih združb Žumberka in Samoborskega gorja. Beta flexible $(\beta=-0.25)$ s Sørensenovim indeksom kot mero razdalje. 
southwestern Europe of the alliance Ilici-Fagion (Mucina et al. 2016) or suballiance Ilici-Fagenion (Willner et al. 2017). Hence this community is considered here as a new association Gentiano asclepiadeae-Fagetum sylvaticae Škvorc, Franjić, Krstonošić et Sever ass. nova hoc loco.

Holotypus hoc loco: Tree layer: Fagus sylvatica 5; Shrub layer: Fagus sylvatica 2, Acer pseudoplatanus +, Frangula alnus +; Herb layer: Vaccinium myrtillus 4, Pteridium aquilinum 2, Gentiana asclepiadea 1, Blechnum spicant 1, Oreopteris limbosperma 1, Frangula alnus 1, Athyrium filixfemina + , Hieracium murorum + , Hieracium rotundatum +, Luzula luzuloides +, Melampyrum pratense +, Prenanthes purpurea +, Rubus hirtus +, Solidago virgaurea +; Moss layer: Leucobryum glaucum 2, Polytrichum formosum 1.

Locality: Right bank of the Presečina stream, Strahinščica, NW Croatia; relevé area $440 \mathrm{~m}^{2}$, elevation 380 $\mathrm{m}$, aspect N-NW; slope $24^{\circ}$, cover tree layer $95 \%$, cover herb layer $75 \%$, date 1975/08/22 (Regula Bevilacqua 1978; Table 35, relevé 3).

The association occurs on silicate bedrock and brown acid soils with a high percentage of raw humus. The soil profile is deeper than the profile supporting other acidophilic beech forests (Regula Bevilacqua 1978, Vukelić 2012). There are many open questions about the definition and distribution of syntaxa within the Luzulo-Fagion alliance in SE Europe (Willner 2002, Vukelić 2012), hence there is a need for a comprehensive numerical analysis of these communities.

Acidophilous beech communities in the researched area are characterized by poor floristic composition, dominated by acidophilous species (Luzula luzuloides, Hieracium murorum, H. racemosum, Vaccinium myrtillus, Melampyrum pratense, Table 1,3$)$. They are mainly distributed at lower altitudes (200-450 m a.s.l.), often on the slopes of deep ditches. Ass. Gentiano asclepiadeae-Fagetum occurs on wetter, more acid and nutrient poorer habitats compared to ass. Luzulo-Fagetum (Figure 3-5).

\section{Ostryo-Fagetum}

Thermophilous beech forests (Ostryo-Fagetum) have also been previously reported in the Samobor highland area (Šugar 1972, Vukelić et al. 2003, Trinajstić \& Cerovečki 2005) and at one part of the Žumberak (Pavletić et al. 1982). These stands occupy warmer habitats with shallow soils on a dolomite base, mostly on ridges or steep southern slopes (Figure 3-5) and often they occur in a mosaic with dry grasslands. The floral composition, besides common beech, is dominated by thermophilic species such as Ostrya carpinifolia, Acer obtusatum, Fraxinus ornus, Sorbus aria, Serratula tinctoria, Carex alba, C. flacca, Peucedanum oreoselinum, Vincetoxicum hirundinaria, Melittis melissophyllum, Tanacetum corymbosum (Table 1). It often represents a transitional stage in a succession of dry grasslands towards the ass. Lamio orvalae-Fagetum, which lasts a very long time due to the harsh environmental conditions. Compared to literature relevés (Pavletić et al. 1982) in new relevés (Table 4) a higher cover of woody species as well as a lower presence of Festuco-Brometea species was registered, which indicates the influence of succession in these stands. The absence of anthropogenic impact on dry grasslands in the studied area (mowing, grazing) leads to a loss of mosaic habitat structure, the reduction in area of thermophilous communities and the homogenization of habitats in general (Erdös et al. 2019).

\section{Vicio oroboidi-Fagetum}

Beech stands considered here as ass. Vicio oroboidi-Fagetum spread in the area of ass. Lamio orvalae-Fagetum stands on less moist, more acidophilic and nutrient poorer habitats (Figure 3,4). Usually this association occurs as extrazonal Illyrian vegetation in the submontane vegetation belt on the southern edge of the Pannonian plain on carbonate bedrock (Vukelić 2012, Marinček \& Čarni 2013) and here it is found on the edge of its distribution. The stands in the studied area are characterized by the absence or poor presence of many typical Aremonio-Fagion species such as Omphalodes verna, Calamintha grandiflora, Aremonia agrimonoides, Rhamnus alpinus ssp. fallax, etc. (Table 1,5), similar to most of the stands in other areas of natural distribution (Vukelić \& Baričević 2002, Marinček \& Čarni 2013).

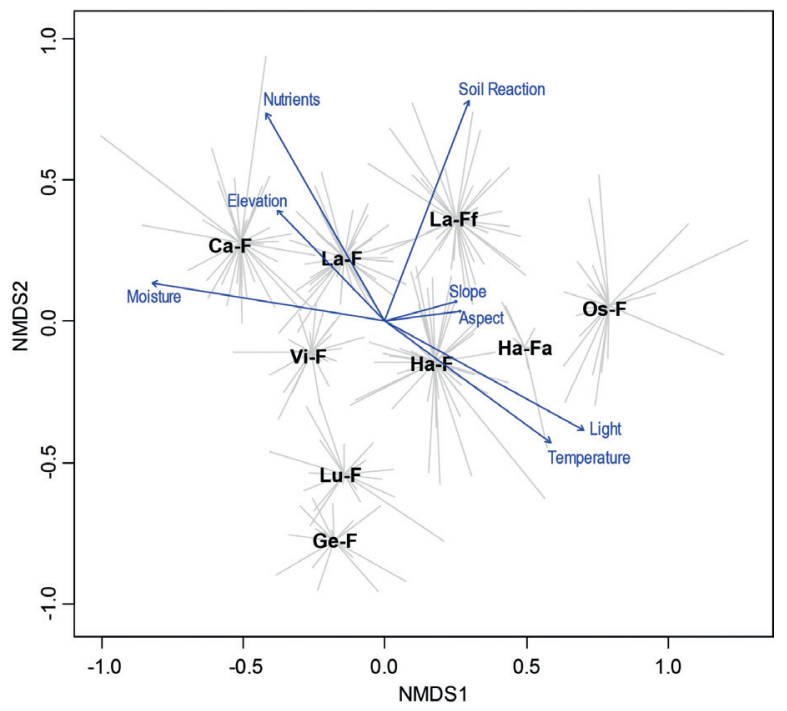

Figure 3: Ordination of the relevés classified into the nine communities based on NMDS of a Bray-Curtis dissimilarity matrix. Community acronimes corresponds to Figure 2.

Slika 3: Ordinacija popisov, razvrščenih v devet združb na osnovi NMDS Bray-Curtisove matrike različnosti. Okrajšave združb so enake kot na Sliki 2. 

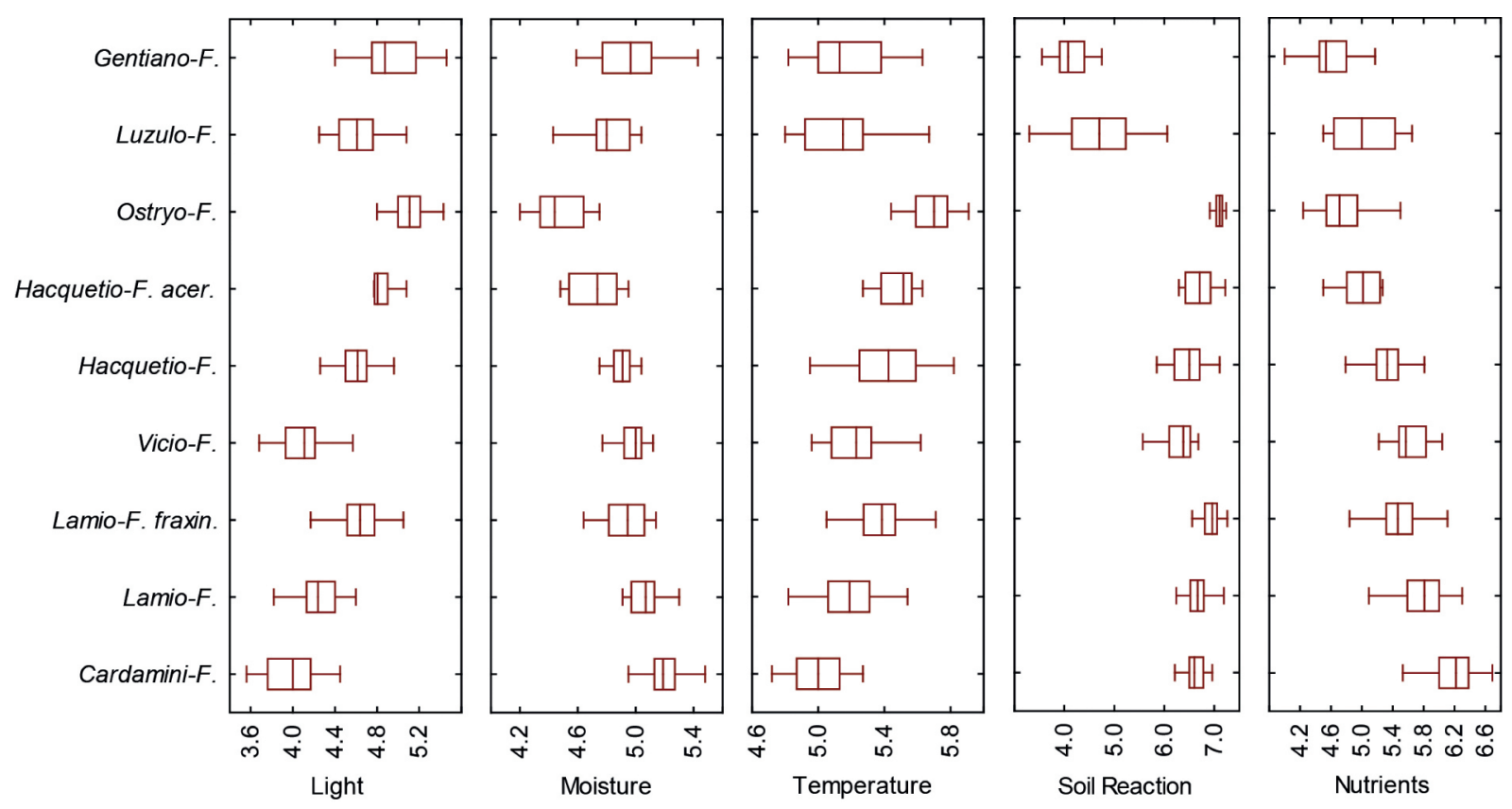

Figure 4: Comparison of analysed beech forest communities based on Ellenberg indicator values. Boxes show the 25-75\% quartile range and the median value; whiskers indicate the range of values, except outliers.

Slika 4: Primerjava analiziranih bukovih gozdnih združb na osnovi Ellenbergovih indikatorski vrednosti. Škatle predstavljajo kvartile (25-75\%) in mediano, ročaji pa prikazujejo razpon vrednosti brez osamelcev.

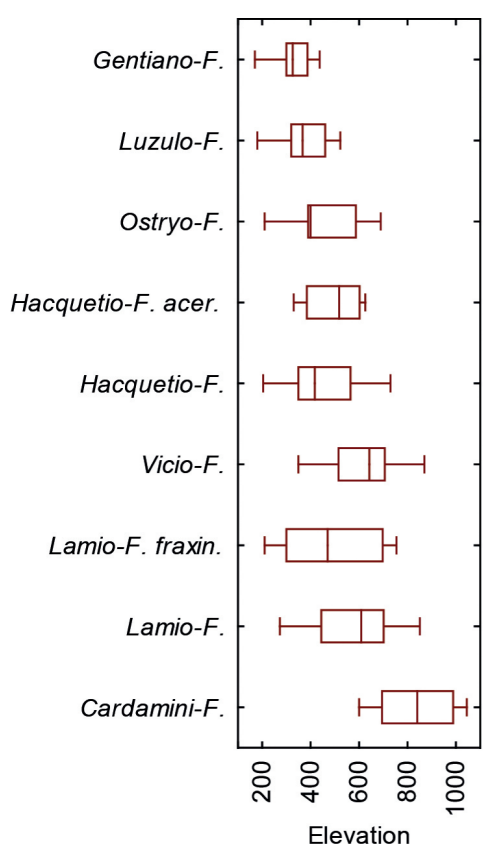

\section{Hacquetio-Fagetum}

The presence of ass. Hacquetio-Fagetum in the Samobor highland area was recorded recently (Trinajstić 2004, Trinajstić \& Pavletić 2004), although it has already been well researched in the neighbouring Gorjanci region

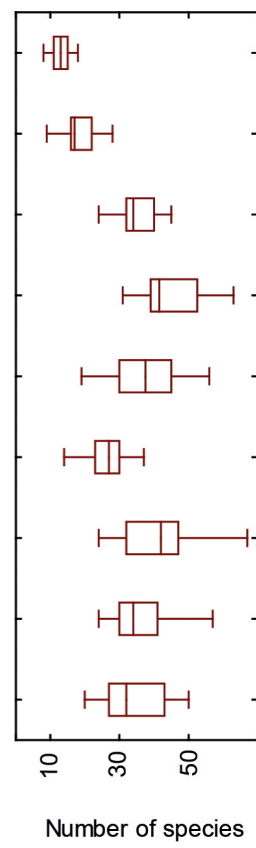

Figure 5: Comparison of analysed beech forest communities based on elevation, aspects transformed according to Beers et al. 1966 (-1.0 NE, 1.0 SW) and number of vascular plant species. Boxes show the $25-75 \%$ quartile range and the median value; whiskers indicate the range of values, except outliers.

Slika 5: Primerjava analiziranih bukovih gozdnih združb na osnovi nadmorske višine, ekspozicije transformirane po Beers et al. 1966 $(-1.0 \mathrm{NE}, 1.0 \mathrm{SW})$ in števila vrst cevnic. Škatle predstavljajo kvartile (25-75\%) in mediano, ročaji pa prikazujejo razpon vrednosti brez osamelcev.

(Košir 1979). It is a widespread community of the preAlpine and pre-Dinaric regions of the Illyrian floral province, in the submontane vegetation belt above sessile oak and hornbeam forests, and below montane beech forests (Vukelić 2012, Marinček \& Čarni 2013). In fact, in the 
study area it usually alternates with ass. Lamio orvalaeFagetum. Ass. Hacquetio-Fagetum spreads on ridges and southern slopes, while ass. Lamio orvalae-Fagetum spreads in ditches and on northern slopes (Figure 5). The tree layer is dominated by Fagus sylvatica, although Quercus petraea and Q. cerris are often present. The shrub layer is rich in species. In the herb layer, Fagetalia species are the most significant (Galium odoratum, Aposeris foetida, Anemone nemorosa, Sanicula europaea, Asarum europaeum, Pulmonaria officinalis etc.). Aremonio-Fagion and EpimedioFagenion species are also well represented - Epimedium alpinum, Hacquetia epipactis, Aremonia agrimonoides, Cyclamen purpurascens, etc. Many thermophilous species are present with a significant abundance - Fraxinus ornus, Sorbus torminalis, S. aria, Viburnum lantana, Carex flacca, Mellytis melyssophyllum, Tanacetum corymbosum, Serratula tinctoria etc. (Table 1, 6).

In the studied area some thermophilous beech stands similar to ass. Ostryo-Fagetum but without Ostrya carpinifolia, are present. These stands are considered here as Hacquetio-Fagetum Ž. Košir ex Borhidi 1963 aceretosum obtusati subass. nova hoc loco (Typus: Table 6, relevé 34 holotypus hoc loco). Ecologically and floristically they are transitional communities between ass. Ostryo-Fagetum and Hacquetio-Fagetum (Figure 3-5, Table 1, 6). These stands are formed by the succession of thermophilous dry grasslands and they are still rich with Festuco-Brometea and Trifolio-Geranietea species (Carex flacca, Cruciata glabra, Tanacetum corymbosum, Solidago virgaurea, Vincetoxicum hirundinaria, etc.). However, they do not occur on a dolomite base and the environmental conditions are more mesophilous. Thus the succession progress of these habitats is faster than in Ostryo-Fagetum, and towards typical Hacquetio-Fagetum.

\section{Lamio orvalae-Fagetum}

It is a widespread community in the studied area and has already been recorded for the Samobor highlands (Vukelić et al. 2003). It occurs between 300 and $800 \mathrm{~m}$ a.s.l, on different slopes, mostly on northern aspects (Figure 5). The habitats are moist, cold and rich in nutrients (Figure 4), usually occurring on limestone and dolomite, and sometimes fragmentary on silicate bedrock (Vukelić et al. 2003). The floral composition is very rich and typical for stands of this association (Vukelić 2012). The tree layer is dominated by Fagus sylvatica with low abundance of other Carpino-Fagetea tree species (most often Acer pseudoplatanus). In the shrub layer there are a lot of typical beech forest species - Fagus sylvatica, Acer pseudoplatanus, A. campestre, A. platanoides, Corylus avellana, Daphne mezereum, Euonymus latifolius, Prunus avium, Sambucus nigra, etc. The herb layer is dominated by Fagetalia spe- cies (Galium odoratum, Carex sylvatica, Mercurialis perennis, Actea spicata, Dentaria bulbifera, Aposeris foetida, Anemone nemorosa, Sanicula europaea, Pulmonaria officinalis), as well as Aremonio-Fagion species (Lamium orvala, Hacquetia epipactis, Vicia oroboides, Cyclamen purpurascens, Aposeris foetida etc.). The ferns (Dryopteris filix-mas, Polystichum aculeatum, P. setiferum etc.) often occur as significant cover (Table 1, 7).

In the area of the Samobor highlands Vukelić et al. (2003) registered a thermophilous variant of the association (var. Acer obtusatum). Similar stands were also found in the Žumberak area and they are common throughout the research area. We consider them here as Lamio orvalae-Fagetum (Horvat 1938) Borhidi 1963 fraxinetosum orni subass. nova hoc loco (Typus: Table 7, relevé 42 holotypus hoc loco). Unlike the typical variant, these stands rarely occur on northern aspects (Figure 5) and they generally occur on warmer habitats (Figure 4). Apart from many mesophilous species characteristic for typical stands, a large number of thermophilous species were also present here (Ostrya carpinifolia, Acer obtusatum, Fraxinus ornus, Viburnum lantana, Rosa arvensis, Carex flacca, Tanacetum corymbosum, etc.). Floristically, it is a transitional community towards ass. Ostryo-Fagetum (Table 1, Figure 3). It could be assumed that these stands present some stage of succession of dry grasslands on dolomite and limestone bedrock abandoned a long time ago, or heavily degraded forest stands which still retain many thermophilous species. According to our results, succession in these stands can be expected to continue towards the typical subassociation and it is doubtful whether on some habitats the stands will stay in a form that will differ from the typical ones.

\section{Cardamino savensi-Fagetum}

Ass. Cardamino savensi-Fagetum is widespread in the higher parts of the researched area (mostly above $700 \mathrm{~m}$ a.s.l., Figure 5). It has not been previously recorded in this area, although it is well researched and documented in the neighbouring area of Gorjanci (Košir 1979, Marinček 1987). It is a zonal association of the highest peaks in the predinaric phytogeographic region on dolomite and limestone bedrock (Marinček \& Čarni 2002) and so far it has been recorded in Croatia only in the highest parts of Papuk (Škvorc et al. 2011). In the research area it is spread on the moistest, coldest and most nutrient rich habitats of all studied communities (Figure 3, 4). Floristically, these stands are similar to stands of ass. Lamio orvalae-Fagetum, but characterized by a greater cover of species indicating more humid and nutrient rich habitats (Acer pseudoplatanus, A. platanoides, Sambucus nigra, Allium ursinum, Dentaria enneaphyllos, Omphalodes verna, etc.) The tree 
layer is dominated by Fagus sylvatica, and very common is Acer pseudoplatanus. The shrub layer is medium to poorly developed. Furthermore, there are many species usual in the montane vegetation belt which differentiate Cardamino savensi-Fagetum from Lamio orvalae-Fagetum - Cardamine trifolia, C. kitaibelli, Sorbus aucuparia, Isopyrum thalictroides, Abies alba, Leucojum vernum, Polygonatum verticillatum, Stellaria nemorum and Dryopteris dilatata (Table 1, 8).

\section{Conclusion}

This is a first systematic study of beech forests in the entire Žumberak and Samobor highland area. There were seven associations and two subassociations recorded, indicating a great diversity of beech communities depending on environmental conditions and anthropogenic influences. Observed succession processes indicate that further homogenization of the habitats will occur in the future, that is, increasing the proportion of mesophilous beech communities at the expense of all kinds of thermophilous forest communities, as well as open grasslands.

\section{Acknowledgements}

This research was financed by Žumberak - Samoborsko gorje Nature Park. We would like to thank Andraž Čarni for substantial advices concerning syntaxonomical nomenclature.

Željko Škvorc DD, https://orcid.org/0000-0002-2848-1454

Daniel Krstonošić $\mathbb{D}$, https://orcid.org/0000-0002-6148-9247

Krunoslav Sever (D), https://orcid.org/0000-0001-8848-1635

\section{References}

Beers, T.W., Dress, P.E. \& Wensel, L. C. 1966: Aspect transformation in site productivity research. Journal of Forestry 64: 691-692.

Borhidi, A. 1960: Fagion-Gesellschaften und Waldtypen im Hügelland von Zselic (Süd-Transdanubien). Ann. Univ. Sci. Budapest. Rolando Eötvös Nornin., Sect. Biol., Budapest 3: 75-87.

Borhidi, A. 1963: Die Zönologie des Verbandes Fagion illyricum. I. Allgemeiner Teil. Acta Bot. Acad. Sci. Hung. 9: 259-297.

Braun-Blanquet, J. 1964: Pflanzensoziologie. Grundzüge der Vegetationskunde. Springer, Wien.

Buzjak, N. 2002: Speleološke pojave u Parku prirode ŽumberakSamoborsko gorje. Geoadria 7: 31-49. https://doi.org/10.15291/ geoadria. 64
Ellenberg, H., Weber, H.E., Düll, R., Wirth, V., Werner, W. \& Paulißen, D. 1992: Zeigerwerte von Pflanzen in Mitteleuropa. Erich Goltze, Göttingen.

Erdös, L., Krstonošić, D., Kiss, P. J., Bátori, Z., Tölgyesi, C. \& Škvorc, Ž. 2019: Plant composition and diversity at edges in a seminatural forest-grassland mosaic. Plant Ecology 220: 279-292. doi. org/10.1007/s11258-019-00913-4.

Hennekens, S.M. \& Schaminée, J.H.J. 2001: TURBOVEG, a comprehensive data base management system for vegetation data. Journal of Vegetation Science 12: 589-591. https://doi. org/10.2307/3237010

Horvat, I. 1929: Rasprostranjenost i prošlost mediteranskih, ilirskih i pontskih elemenata u flori sjeverne Hrvatske i Slovenije. Acta Botanica Croatica 4: 1-34.

Horvat, I. 1938: Biljnosociološka istraživanja šuma u Hrvatskoj. Glasnik za Šumske Pokuse 6: 127-279.

Horvat, I. 1950: Šumske zajednice Jugoslavije. Institut za šumarska istraživanja NR Hrvatske. Zagreb.

Horvat, I. 1962: Vegetacija planina zapadne Hrvatske. Prir. Istraž. Jugosl. Akad. 30: 3-179.

Jelaska, S.D., Kušan, V., Peternel, H., Grgurić, Z., Mihulja, A. \& Major, Z. 2005: Vegetation mapping of Zumberak - Samoborsko gorje Nature Park, Croatia, using Landsat 7 and field data. Acta Botanica Croatica 64: 303-311.

Košir, Ž. 1979: Ekološke in fitocenološke razmere v gorskem in hribovitem jugozahodnem obrobju Panonije. Zveza gozdarskih društev Slovenije, Ljubljana. pp. 149.

Marinček, L. 1970: Bukov gozd z rebrenjačo (Blechno-Fagetum). Zbornik Biotehniške fakultete v Ljubljani (Ljubljana): 93-130.

Marinček, L. 1987: Prispevek k poznavanju acidofilnih gozdov belega gabra Slovenije. Razprave IV. razreda SAZU 27: 65-99.

Marinček, L. \& Čarni, A. 2002: Commentary to the vegetation map of forest communities of slovenia in a scale of 1:400,000. Biološki inštitut Jovana Hadžija ZRC SAZU.

Marinček, L. \& Čarni, A. 2013: Submontanski bukovi gozdovi podzveze Epimedio-Fagenion (Aremonio-Fagion). Scopolia 78: 1-75.

Marinček, L. \& Zupančić, M. 1995: Nomenklaturna revizija acidofilnih bukovih in gradnovih gozdov zahodnega območja ilirske florne province. Hladnikia 4: 29-35.

Marinček, L., Košir, P. \& Šilc, U. 2001: Prispevek k sinsistematiki asociacije Isopyro-Fagetum Košir 1962. Hladnikia 12: 41-46.

Marinček, L., Mucina, L., Zupančič, M., Poldini, L., Dakskobler, I. \& Accetto, M. 1993: Nomenklatorische Revision der illyrischen Buchenwälder. Studia Geobotanica 12: 121-135.

McCune, B. \& Mefford, M.J. 2006: PC-ORD. Multivariate Analysis of Ecological Data. Version 5.10 MjM Software, Gleneden Beach, Oregon, U.S.A.

Mucina, L., Bültmann, H., Dierßen, K., Theurillat, J.-P., Raus, T., Čarni, A., ... \& Tichý, L. 2016: Vegetation of Europe: hierarchical floristic classification system of vascular plant, bryophyte, lichen, and algal communities. Applied Vegetation Science 19 (Suppl. 1): 3-264. DOI: https://doi.org/10.1111/avsc. 12257 
Nikolić, T., (ed.) 2020: Flora Croatica baza podataka. On-Line (http:// hirc.botanic.hr/fcd). Botanički zavod, Prirodoslovno-matematički fakultet, Zagreb.

Pamić, J. \& Tomljenović, B. 1998: Basic geologic data from the Croatian part of the Zagorje- Mid-Transdanubian Zone. Acta Geologica Hungarica 41: 389-400.

Pavletić, Zi., Trinajstić, I. \& Šugar, I. 1982: Die wärmeliebenden Hopfenbuchen-Buchenwälder (Ostryo-Fagetum Wraber) in NordwestKroatien. Studia Geobotanica 2: 15-19.

Penzar, B. \& Penzar, I. 1982: Prikaz godišnjeg hoda oborina u Hrvatskoj pomoću Köppenove sheme. Radovi (Šumarski institut) 17/18: 3-9.

Regula-Bevilacqua, Lj. 1978: Biljni pokrov Strahinščice u Hrvatskom Zagorju. PhD thesis. Faculty of Sciences, University of Zagreb.

Rivas-Martinez, S. 1962: Contribuciön al estudo fitosociolögico de los hayelas espanolas. Anales Inst. Bot. Cavanilles 20: 97-128.

Statsoft, Inc., 2005: STATISTICA (data analysis software system), version 7.1 .

Šilc, U. \& Čarni, A. 2012: Conspectus of vegetation syntaxa in Slovenia. Hacquetia 11: 113-164. DOI: https://doi.org/10.2478/ v10028-012-0006-1

Škvorc, Ž., Franjić, J., Krstonošić, D., Sever, K., Alešković, I. 2011: Vegetacijska obilježja bukovih šuma Psunja, Papuka i Krndije. Croatian Journal of Forest Engineering 32: 157-176.

Škvorc, Ž., Jasprica, N., Alegro, A., Kovačić, S., Franjić, J., Krstonošić, D., Vraneša, A. \& Čarni, A. 2017: Vegetation of Croatia: Phytosociological classification of the high-rank syntaxa. Acta Botanica Croatica 76: 200-224. DOI: https://doi.org/10.1515/ botcro-2017-0014

Šugar, I. 1972: Biljni svijet Samoborskog gorja. PhD thesis. Faculty of Sciences, University of Zagreb.

Theurillat, J.-P., Willner, W., Fernández-González, F., Bültmann, H., Čarni, A., Gigante, G., Mucina, L., \& Weber, H. 2020: International Code of Phytosociological Nomenclature. 4th edition. Applied Vegetation Science. DOI: https://doi.org/10.1111/avsc. 12491

Tichý, L. \& Chytrý, M. 2006: Statistical determination of diagnostic species for site groups of unequal size. Journal of Vegetation Science 17: 809-818. DOI: https://doi.org/10.1111/j.1654-1103.2006. tb02504.x

Tichý, L. 2002: JUICE, software for vegetation classification. Journal of Vegetation Science 13: 451-453. DOI: https://doi. org/10.1111/j.1654-1103.2002.tb02069.x

Tichý, L., Chytrý, M., Hájek, M., Talbot, S.S. \& Botta-Dukát, Z. 2010: OptimClass: using species-to-cluster fidelity to determine the optimal partition in classification of ecological communities. Journal of Vegetation Science 21: 287-299. DOI: https://doi.org/10.1111/ j.1654-1103.2009.01143.x

Trinajstić, I. 1995: Samoborsko gorje, a refuge of various floral elements between the Alps and the Dinaric mountains. Acta Botanica Croatica 54: 47-72.

Trinajstić, I. 2004: Fitocenološko-sintaksonomska analizi asocijacije Hacquetio-Fagetum Košir (1962) 1979 (Aremonio-Fagion) u vegetaciji Hrvatske. Šumarski list 128: 3-11.
Trinajstić, I., 2004: Nomenklaturno-sintaksonomska revizija kompleksa “Blechno-Fagetum (Ht. 1950) Marinček 1970” (LuzuloFagion). Šumarski list 128: 375-380.

Trinajstić, I. 2008: Biljne zajednice Republike Hrvatske. Akademija šumarskih znanosti, Zagreb.

Trinajstić, I. \& Cerovečki Z. 2005: Prilog sintaksonomskoj analizi asocijacije Ostryo-Fagetum (M. Wraber) ex Trinajstić 1972. Šumarski list 129: 575-581.

Trinajstić, I. \& Pavletić, Zi. 2004: The association Hacquetio-Fagetum Košir 1962 (Aremonio-Fagion) in Croatia. Hacquetia 3 (2): 29-42.

Tüxen, R. \& Oberdorfer, E. 1958: Die Pflanzenwelt Spaniens. Ergebnisse der 10. Internationalen Pflanzengeographischen Exkursion (IPE) durch Spanien 1953. II. Teil. Eurosibirische PhanerogamenGesellschaften Spaniens mit Ausblick auf die Alpine- und die Mediterran-Region dieses Landes. Veröff. Geobot. Inst. Rübel Zürich 32, Hans Huber, Bern.

Vrbek, M. 2005: Flora i nešumska vegetacija Žumberka. PhD thesis. Faculty of Sciences, University of Zagreb.

Vrbek, M. 2010: Žumberak. In: Nikolić, T., Topić, J. \& Vuković, N. (Eds.). Botanički važna područja Hrvatske. Prirodoslovno-matematički fakultet, Školska knjiga Zagreb.

Vukelić, J. 2012: Šumska vegetacija Hrvatske. Šumarski fakultet Sveučilišta u Zagrebu, Državni zavod za zaštitu prirode. Zagreb.

Vukelić, J. \& Baričević, D. 2002: Novije fitocenološke spoznaje o bukovim šumama u Hrvatskoj. Šumarski list 126: 439-457.

Vukelić, J., Baričević, D. \& Drvenkar, D. 2003: Fitocenološke karakteristike bukovih šuma u Samoborskom gorju. Šumarski list 127: 531-544.

Westhoff V. \& Maarel E. van der. 1973: The Braun-Blanquet Approach. In: Whittaker, R.H. (ed.) Ordination and Classification of Communities: 617-726. W. Junk, The Hague.

Willner, W. 2002: Syntaxonomische Revision der südmitteleuropäischen Buchenwälder. Phytocoenologia 32: 337-453.

Willner, W., Jiménez-Alfaro, B., Agrillo, E., Biurrun, I., Campos, J. A. Čarni, A., Casella, L., Csiky, J., Ćušterevska, R., Didukh, Y. P., Ewald, J., Jandt, U., Jansen, F., Kącki, Z., Kavgaci, A., Lenoir, J., Marinšek, A., Onyshchenko, V., Rodwell, J., Schaminée, J., Šibík, J., Škvorc, Ž., Svenning, J.-C., Tsiripidis, I., Turtureanu, P. D., Tzonev, R., Vassilev, K., Venanzoni, R., Wohlgemuth, T., Chytrý, M. 2017: Classification of European beech forests: a Gordian Knot? Applied Vegetation Science 20: 494-512. DOI: https://doi.org/10.1111/avsc.12299

Zaninović, K., Srnec, L. \& Perčec Tadić, M. 2004: Digitalna godišnja temperaturna karta Hrvatske (Digital annual temperature map of Croatia). Hrvatski meteorološki časopis 39: 51-58.

Zelený, D. \& Schaffers, A. P. 2012: Too good to be true: pitfalls of using mean Ellenberg indicator values in vegetation analyses. Journal of Vegetation Science 23: 419-431. DOI: https://doi.org/10.1111/ j.1654-1103.2011.01366.x 
Table 1: Synoptic tables of analysed communities of beech forests (including literature relevés). Only species with phi values higher than 0.3 and a frequency higher than $30 \%$ in at least one group are included. Grey shaded values indicate diagnostic species with high $(\Phi \geq 0.3)$ fidelity to particular clusters. At the end of the table frequencies of additional most abundant species are shown (frequency higher than $60 \%$ in at least one group). Community acronimes corresponds to Figure 2.

Tabela 1: Sinoptična tabela preučevanih združb bukovih gozdov (vključno s popisi iz literature). Prikazane so samo vrste $s$ fi vrednostjo večjo od 0,3 in frekvenco višjo od $30 \%$ v vsaj eni skupini. Sivo zasenčeno so diagnostične vrste z večjo $(\Phi \geq 0,3)$ navezanostjo na določen klaster. Na koncu tabele so prikazane najbolj pogoste vrste s frekvencami (frekvenca višja od 60 \% vsaj eni skupini). Okrajšave združb so enake kot v Sliki 2.

\begin{tabular}{|c|c|c|c|c|c|c|c|c|c|c|}
\hline Community & & $\mathrm{Ca}-\mathrm{F}$ & Vi-F & $\mathrm{La}-\mathrm{F}$ & La-Ff & $\mathrm{Ha}-\mathrm{F}$ & $\mathrm{Ha}-\mathrm{Fa}$ & Os-F & $\mathrm{Lu}-\mathrm{F}$ & Ge-F \\
\hline Number of relevés & Layer & 39 & 21 & 39 & 36 & 42 & 8 & 21 & 19 & 18 \\
\hline Sambucus nigra & B & 74 & 5 & 28 & 6 & 2 & . & . & 5 & . \\
\hline Arum maculatum & $\mathrm{C}$ & 64 & 5 & 23 & . & . & . & . & . & . \\
\hline Cardamine kitaibelli & $\mathrm{C}$ & 59 & 5 & 13 & 3 & . & . & . & . & . \\
\hline Lonicera alpigena & $\mathrm{B}$ & 54 & . & 13 & 3 & 2 & . & . & . & . \\
\hline Paris quadrifolia & $\mathrm{C}$ & 72 & 10 & 26 & 14 & 10 & . & . & . & . \\
\hline Geranium robertianum & $\mathrm{C}$ & 36 & . & 3 & . & . & . & . & . & . \\
\hline Cardamine trifolia & $\mathrm{C}$ & 51 & 5 & 8 & 8 & 2 & . & . & . & . \\
\hline Isopyrum thalictroides & $\mathrm{C}$ & 31 & . & 3 & 3 & . & . & . & . & . \\
\hline Ranunculus lanuginosus & $\mathrm{C}$ & 38 & 10 & 8 & 3 & . & . & . & . & . \\
\hline Senecio ovatus & $\mathrm{C}$ & 54 & 5 & 23 & 31 & 5 & . & . & 5 & . \\
\hline Oxalis acetosella & $\mathrm{C}$ & 31 & 5 & 5 & 3 & 2 & . & . & . & . \\
\hline Dryopteris filix-mas & $\mathrm{C}$ & 92 & 67 & 74 & 44 & 33 & 13 & . & 11 & . \\
\hline Asplenium scolopendrium & $\mathrm{C}$ & 41 & 10 & 15 & 8 & 2 & $\cdot$ & 5 & . & . \\
\hline Polygonatum multiflorum & $\mathrm{C}$ & 72 & 43 & 46 & 28 & 17 & . & 5 & 11 & . \\
\hline Dentaria enneaphyllos & $\mathrm{C}$ & 41 & . & 18 & 19 & 5 & . & . & . & . \\
\hline Circaea lutetiana & $\mathrm{C}$ & 31 & . & 8 & 3 & 5 & . & . & 5 & . \\
\hline Dentaria bulbifera & $\mathrm{C}$ & 87 & 71 & 64 & 31 & 38 & 13 & 5 & 16 & . \\
\hline Acer pseudoplatanus & A & 74 & 10 & 64 & 39 & 31 & 25 & 14 & . & . \\
\hline Anemone nemorosa & $\mathrm{C}$ & 64 & 71 & 54 & 8 & 29 & 13 & . & 11 & . \\
\hline Actaea spicata & $\mathrm{C}$ & 56 & 33 & 67 & 36 & 19 & . & 5 & . & . \\
\hline Polystichum aculeatum & $\mathrm{C}$ & 33 & 14 & 41 & 8 & 5 & . & . & . & . \\
\hline Lamium orvala & $\mathrm{C}$ & 51 & 38 & 64 & 50 & 29 & $\cdot$ & . & . & . \\
\hline Cornus sanguinea & $\mathrm{B}$ & . & . & 18 & 72 & 40 & 38 & 5 & . & . \\
\hline Epimedium alpinum & $\mathrm{C}$ & 3 & 52 & 13 & 14 & 62 & 13 & 19 & 11 & . \\
\hline Vincetoxicum hirundinaria & $\mathrm{B}$ & . & . & . & 22 & 5 & 63 & 29 & . & . \\
\hline Knautia drymeia & $\mathrm{C}$ & 3 & . & 10 & 22 & 19 & 75 & 48 & 5 & . \\
\hline Ostrya carpinifolia & A & . & 5 & 18 & 42 & 12 & 25 & 95 & 5 & . \\
\hline Peucedanum oreoselinum & $\mathrm{C}$ & . & . & . & 3 & . & 13 & 52 & . & . \\
\hline Sorbus aria & A & . & . & . & 28 & 7 & . & 67 & . & 6 \\
\hline Mercurialis ovata & $\mathrm{C}$ & 3 & . & . & 8 & 5 & 13 & 52 & . & . \\
\hline Melittis melissophyllum & $\mathrm{C}$ & . & . & 8 & 25 & 36 & 63 & 90 & 5 & . \\
\hline Clematis recta & $\mathrm{C}$ & . & . & . & 6 & . & . & 33 & . & . \\
\hline Chamaecytisus hirsutus & $\mathrm{B}$ & . & . & . & 3 & 5 & 13 & 43 & 5 & . \\
\hline Polygonatum odoratum & $\mathrm{C}$ & . & . & 10 & 17 & 5 & $\cdot$ & 43 & . & . \\
\hline Carex flacca & $\mathrm{C}$ & 3 & 14 & . & 50 & 24 & 75 & 81 & . & . \\
\hline Quercus pubescens & A & . & . & . & . & . & 13 & 33 & . & . \\
\hline Campanula persicifolia & $\mathrm{C}$ & . & . & 3 & 19 & 14 & 38 & 52 & . & . \\
\hline Berberis vulgaris & B & . & . & 3 & 19 & 12 & 13 & 43 & . & . \\
\hline Rhamnus cathartica & $\mathrm{B}$ & . & . & 3 & 19 & 2 & 13 & 38 & . & . \\
\hline Sorbus torminalis & B & . & 10 & 8 & 19 & 21 & 50 & 71 & 26 & 33 \\
\hline Fraxinus ornus & $\mathrm{B}$ & 5 & 5 & 21 & 75 & 74 & 75 & 95 & 32 & 22 \\
\hline
\end{tabular}




\begin{tabular}{|c|c|c|c|c|c|c|c|c|c|c|}
\hline Community & & $\mathrm{Ca}-\mathrm{F}$ & $\mathrm{Vi}-\mathrm{F}$ & $\mathrm{La}-\mathrm{F}$ & La-Ff & $\mathrm{Ha}-\mathrm{F}$ & $\mathrm{Ha}-\mathrm{Fa}$ & Os-F & $\mathrm{Lu}-\mathrm{F}$ & Ge-F \\
\hline Number of relevés & Layer & 39 & 21 & 39 & 36 & 42 & 8 & 21 & 19 & 18 \\
\hline Convallaria majalis & C & . & 5 & 13 & 19 & 31 & 5 & 62 & 16 & 11 \\
\hline Sorbus aria & $\mathrm{B}$ & 8 & . & 15 & 28 & 19 & 38 & 57 & 16 & 6 \\
\hline Viburnum lantana & $\mathrm{B}$ & . & . & 21 & 58 & 29 & 63 & 67 & 5 & . \\
\hline Cephalanthera rubra & $\mathrm{C}$ & . & . & 5 & 3 & 5 & 13 & 33 & . & 6 \\
\hline Hieracium murorum & $\mathrm{C}$ & . & 1 & 8 & 3 & 21 & 38 & 5 & 74 & 28 \\
\hline Blechnum spicant & $\mathrm{C}$ & . & . & . & . & . & . & . & . & 67 \\
\hline Frangula alnus & $\mathrm{B}$ & . & . & . & . & 2 & . & . & 5 & 44 \\
\hline Calluna vulgaris & $\mathrm{C}$ & . & . & . & . & . & . & . & 21 & 44 \\
\hline Pteridium aquilinum & $\mathrm{C}$ & 3 & 43 & 28 & 33 & 74 & 63 & 5 & 63 & 89 \\
\hline Quercus petraea & B & . & 19 & 3 & 3 & 33 & 5 & 5 & 42 & 61 \\
\hline Athyrium filix-femina & $\mathrm{C}$ & 67 & 62 & 28 & 8 & 7 & . & . & 32 & . \\
\hline Acer obtusatum & A & . & . & 18 & 58 & 21 & 88 & 90 & . & . \\
\hline Fraxinus ornus & A & . & . & 3 & 19 & 5 & 63 & 57 & . & . \\
\hline Serratula tinctoria & $\mathrm{C}$ & . & . & 3 & 14 & 29 & 75 & 62 & 16 & 17 \\
\hline Luzula luzuloides & $\mathrm{C}$ & 18 & 48 & 13 & 6 & 36 & 25 & 5 & 100 & 94 \\
\hline Vaccinium myrtillus & B & . & . & . & . & . & . & . & 63 & 94 \\
\hline Castanea sativa & B & . & 38 & 3 & 8 & 38 & . & . & 79 & 89 \\
\hline Castanea sativa & A & . & 29 & 5 & 3 & 36 & 25 & 5 & 68 & 78 \\
\hline Quercus petraea & A & . & 38 & 10 & 25 & 79 & 75 & 38 & 63 & 83 \\
\hline \multicolumn{11}{|l|}{ Tree layer } \\
\hline Fagus sylvatica & & 100 & 100 & 100 & 100 & 100 & 100 & 100 & 100 & 100 \\
\hline \multicolumn{11}{|l|}{ Shrub layer } \\
\hline Fagus sylvatica & & 100 & 95 & 95 & 86 & 100 & 100 & 67 & 95 & 89 \\
\hline Acer campestre & & 13 & 24 & 31 & 50 & 60 & 50 & 29 & 11 & . \\
\hline Tamus communis & & 8 & 19 & 64 & 56 & 45 & 63 & 52 & . & . \\
\hline Rubus hirtus & & 67 & 76 & 85 & 33 & 62 & 25 & 5 & 63 & 44 \\
\hline Acer pseudoplatanus & & 69 & 57 & 77 & 61 & 45 & 63 & 24 & 16 & . \\
\hline Daphne mezereum & & 41 & 24 & 64 & 64 & 50 & 63 & 52 & . & . \\
\hline Rosa arvensis & & 21 & 19 & 31 & 58 & 50 & 63 & 48 & . & . \\
\hline \multicolumn{11}{|l|}{ Herb layer } \\
\hline Melampyrum pratense & & 3 & 5 & 21 & 19 & 38 & 38 & 29 & 68 & 67 \\
\hline Helleborus niger & & 23 & 5 & 23 & 36 & 19 & 63 & 24 & . & . \\
\hline Tanacetum corymbosum & & 3 & . & 8 & 42 & 38 & 75 & 62 & 11 & . \\
\hline Galium odoratum & & 9 & 86 & 77 & 36 & 57 & 38 & 24 & 26 & . \\
\hline Gentiana asclepiadea & & 18 & 38 & 44 & 39 & 64 & 63 & 5 & 47 & 67 \\
\hline Salvia glutinosa & & 10 & 24 & 38 & 50 & 43 & 50 & 62 & . & . \\
\hline Pulmonaria officinalis & & 31 & 71 & 62 & 67 & 74 & 38 & 38 & 5 & . \\
\hline Cyclamen purpurascens & & 46 & 57 & 77 & 67 & 60 & 75 & 43 & 5 & . \\
\hline Lathyrus vernus & & . & 14 & 46 & 44 & 36 & 63 & 33 & . & . \\
\hline Potentilla micrantha & & 13 & 14 & 15 & 25 & 31 & 63 & 24 & . & . \\
\hline Solidago virgaurea & & 3 & 1 & 5 & 56 & 38 & 63 & 43 & 47 & 28 \\
\hline Asarum europaeum & & 5 & 19 & 46 & 61 & 52 & 50 & 10 & 5 & . \\
\hline Mercurialis perennis & & 56 & 19 & 64 & 64 & 19 & 25 & 14 & . & . \\
\hline Aposeris foetida & & 23 & 52 & 38 & 61 & 69 & 50 & 57 & 58 & 6 \\
\hline Galium sylvaticum & & 8 & 33 & 26 & 61 & 60 & 75 & 62 & 32 & 6 \\
\hline Carex sylvatica & & 64 & 67 & 64 & 50 & 43 & 25 & 19 & 5 & . \\
\hline Hedera helix & & 44 & 43 & 77 & 58 & 64 & 88 & 52 & 42 & 6 \\
\hline Sanicula europaea & & 21 & 71 & 56 & 61 & 67 & 50 & 24 & 42 & 6 \\
\hline Prenanthes purpurea & & 46 & 43 & 33 & 14 & 21 & . & . & 63 & 61 \\
\hline
\end{tabular}

Vegetation layers: A - tree layer, B - shrub layer, C - herb layer 
Table 2: Spearman correlations of first two NMDS axes with environmental variables. Only significant correlations are presented $(\mathrm{p}<0.01)$.

Tabela 2: Spearmanove korelacije prvih dveh osi NMDS z okoljskimi spremenljivkami. Prikazane so samo statistično značilne korelacije $(\mathrm{p}<0,01)$.

\begin{tabular}{lcc}
\hline Environmental variable & Axis1 & Axis2 \\
Elevation & 0.53 & 0.18 \\
Light EIV & 0.88 & \\
Temperature EIV & 0.75 & \\
Moisture EIV & 0.84 & \\
Soil Reaction EIV & & 0.85 \\
Nutrients EIV & 0.81 & 0.31 \\
\hline
\end{tabular}

Table 3 (Tabela 3): Ass. Luzulo luzuloidi-Fagetum.

\begin{tabular}{lccccc}
\hline Relevé No. & 1 & 2 & 3 & 4 & 5 \\
\hline Date 2010 (Day/Month) & $26 / 5$ & $21 / 5$ & $21 / 5$ & $9 / 7$ & $29 / 6$ \\
Elevation (m) & 523 & 680 & 687 & 508 & 381 \\
Aspect $\left(^{\circ}\right)$ & 135 & 200 & 250 & 304 & 35 \\
Slope $\left(^{\circ}\right)$ & 10 & 15 & 50 & 50 & 8 \\
Cover of layers (\%): & & & & & \\
Tree (high) & 90 & 100 & 100 & 90 & 100 \\
Tree (middle) & 40 & 10 & 10 & 10 & 10 \\
Shrub & 15 & 5 & 10 & 10 & 2 \\
Herb & 40 & 50 & 80 & 60 & 10 \\
\hline
\end{tabular}

Charact. species of the ass.

Luzula luzuloides

Hieracium racemosum

\section{Luzulo-Fagion}

Vaccinium myrtillus

Melampyrum pratense

Polypodium vulgare

Fagetalia

Fagus sylvatica

Fagus sylvatica

Fagus sylvatica

Fagus sylvatica

Aposeris foetida

Cardamine bulbifera

Sanicula europaea

Epimedium alpinum

Prenanthes purpurea

Galium odoratum

Lamium galeobdolon

Polygonatum multiflorum

Cyclamen purpurascens

Vicia oroboides

Asarum europaeum

\begin{tabular}{|c|c|c|c|c|c|c|}
\hline Relevé No. & & 1 & 2 & 3 & 4 & 5 \\
\hline Euphorbia dulcis & & + & . & . & . & $\cdot$ \\
\hline Festuca altissima & & . & . & . & + & . \\
\hline Pulmonaria officinalis & & . & . & . & + & . \\
\hline Homogyne sylvestris & & . & . & . & + & $\cdot$ \\
\hline Athyrium filix-femina & & . & + & . & . & - \\
\hline \multicolumn{7}{|l|}{ Carpino-Fagetea } \\
\hline Quercus petraea & a1 & 2 & . & . & + & 2 \\
\hline Quercus petraea & $\mathrm{b}$ & . & . & . & . & + \\
\hline Prunus avium & a1 & + & . & . & . & . \\
\hline Prunus avium & $\mathrm{b}$ & + & . & . & . & . \\
\hline Acer pseudoplatanus & & . & + & 2 & . & + \\
\hline Sorbus aria & & + & . & . & + & . \\
\hline Ilex aquifolium & & . & . & . & 1 & . \\
\hline Hieracium murorum & c & . & + & + & 1 & + \\
\hline Anemone nemorosa & & 1 & . & + & . & . \\
\hline Hedera helix & & . & + & . & + & . \\
\hline Luzula pilosa & & . & . & . & . & 1 \\
\hline Carex pilosa & & . & . & . & + & . \\
\hline Convallaria majalis & & + & . & . & . & . \\
\hline Knautia drymeia & & . & . & + & . & . \\
\hline Melica nutans & & + & . & . & . & . \\
\hline Carex digitata & & . & . & . & + & . \\
\hline Galium sylvaticum & & . & . & + & . & . \\
\hline Galium schultesii & & . & . & . & + & . \\
\hline Symphytum tuberosum & & . & + & . & . & . \\
\hline
\end{tabular}

Quercetea roboris

Castanea sativa

Castanea sativa

Castanea sativa

Castanea sativa

Betula pendula

Quercetea pubescentis

Fraxinus ornus

Sorbus torminalis

Acer obtusatum

\section{Rhamno-Prunetea}

Rubus hirtus

Corylus avellana

Juniperus communis

\section{Trifolio-Geranietea}

Chamaecytisus hirsutus

Solidago virgaurea

Tanacetum corymbosum

\section{Festuco-Brometea}

Cruciata glabra

Dorycnium germanicum

Molinio-Arrbenatheretea

Gentiana asclepiadea

Platanthera bifolia 


\begin{tabular}{|c|c|c|c|c|c|c|}
\hline Relevé No. & & 1 & 2 & 3 & 4 & 5 \\
\hline Ajuga reptans & & . & . & . & . & + \\
\hline \multicolumn{7}{|l|}{ Other species } \\
\hline Pteridium aquilinum & c & + & + & . & + & + \\
\hline Clematis vitalba & & . & + & . & . & . \\
\hline
\end{tabular}

\section{Legend:}

a1 - High tree layer,

a2 - Middle tree layer,

b - Shrub layer,

c - Herb layer.

Coordinates of the relevés:

$\begin{array}{lll}\mathbf{1} 45.72941315 .406087 ; & \mathbf{2} 45.82007215 .5198456 ; \\ \mathbf{3} 45.816549415 .5144942 ; & \mathbf{4} 45.74383515 .66879 ;\end{array}$

545.673940715 .4423374

Table 4 (Tabela 4): Ass. Ostryo-Fagetum.

\begin{tabular}{lcccc}
\hline Relevé No. & 1 & 2 & 3 & 4 \\
\hline Date 2010 (Day/Month) & $21 / 5$ & $9 / 7$ & $9 / 7$ & $9 / 7$ \\
Elevation (m) & 524 & 690 & 686 & 677 \\
Aspect $\left(^{\circ}\right)$ & 75 & 339 & 163 & 231 \\
Slope $\left(^{\circ}\right)$ & 65 & 35 & 40 & 30 \\
Cover of layers (\%): & & & & \\
Tree (high) & 100 & 90 & 100 & 90 \\
Tree (middle) & 30 & 20 & 20 & 40 \\
Shrub & 10 & 10 & 10 & 10 \\
Herb & 70 & 20 & 40 & 50 \\
\hline
\end{tabular}

\section{Charact. species of the ass.}

Ostrya carpinifolia

Ostrya carpinifolia

Ostrya carpinifolia

Ostryo-Fagenion

Acer obtusatum

Acer obtusatum

Acer obtusatum

Fraxinus ornus

Fraxinus ornus

Mercurialis ovata

Aremonio-Fagion

Aposeris foetida

Knautia drymeia

Aremonia agrimonoides

Helleborus dumetorum

Homogyne sylvestris

\section{Fagetalia}

Fagus sylvatica

Fagus sylvatica

Fagus sylvatica

Daphne mezereum

Primula vulgaris

\begin{tabular}{lcccc}
\hline Relevé No. & 1 & 2 & 3 & 4 \\
Mycelis muralis &. & + & + &. \\
Mercurialis perennis &. & + & + &. \\
Polygonatum multiflorum & + &. &. &. \\
Cardamine bulbifera & + &. &. &. \\
Lathyrus vernus &. & + &. &. \\
Pulmonaria officinalis & + &. &. &. \\
Campanula trachelium &. &. & + &. \\
Brachypodium sylvaticum &. & + &. &. \\
Festuca altissima &. & + &. &.
\end{tabular}

\section{Carpino-Fagetea}

Quercus petraea

Carpinus betulus

Rosa arvensis

Convallaria majalis

Melittis melissophyllum

Hedera helix

Melica nutans

Galium sylvaticum

Lathyrus niger

Hieracium murorum

Carex digitata

Cephalanthera damasonium

Cephalanthera rubra

\section{Quercetea pubescentis}

Sorbus aria

Sorbus aria

Sorbus aria

Quercus cerris

Amelanchier ovalis

Sorbus torminalis

Tamus communis

Viburnum lantana

Genista tinctoria

Cephalanthera longifolia

Festuca heterophylla

Vincetoxicum hirundinaria

Clinopodium vulgare

Quercetea roboris

Serratula tinctoria

Luzula luzuloides

Erico-Pinetea

Carex alba

Rhamno-Prunetea

Berberis vulgaris

Juniperus communis

Viburnum opulus

Rhamnus cathartica

Epilobietea angustifolii

Salvia glutinosa

Heracleum sphondylium

Trifolio-Geranietea 


\begin{tabular}{|c|c|c|c|c|}
\hline Relevé No. & & 1 & 2 & 3 \\
\hline Chamaecytisus hirsutus & $\mathrm{b}$ & + & . & . \\
\hline Tanacetum corymbosum & c & . & + & + \\
\hline Peucedanum oreoselinum & & + & . & + \\
\hline Solidago virgaurea & & + & + & . \\
\hline Campanula persicifolia & & + & . & + \\
\hline Iris graminea & & . & + & + \\
\hline Clematis recta & & . & . & + \\
\hline Veronica chamaedrys & & + & . & . \\
\hline Silene nutans & & . & . & . \\
\hline Potentilla micrantha & & . & . & + \\
\hline Buphthalmum salicifolium & & . & . & . \\
\hline \multicolumn{5}{|l|}{ Mulgedio-Aconitetea } \\
\hline Thalictrum aquilegiifolium & c & + & + & . \\
\hline Phyteuma ovatum & & . & + & . \\
\hline Cirsium erisithales & & . & + & - \\
\hline Centaurea montana & & & + & \\
\hline
\end{tabular}

Festuco-Brometea

Carex flacca

Cruciata glabra

\begin{tabular}{|c|c|c|c|c|c|}
\hline Relevé No. & & 1 & 2 & 3 & 4 \\
\hline Anthericum ramosum & & . & . & + & + \\
\hline Viola hirta & & . & . & + & + \\
\hline Euphorbia cyparissias & & . & . & . & + \\
\hline Pimpinella saxifraga & & $\mathrm{r}$ & . & . & \\
\hline \multicolumn{6}{|l|}{ Other species } \\
\hline Clematis vitalba & $\mathrm{b}$ & . & + & . & \\
\hline Malus sylvestris & & + & . & . & \\
\hline Platanthera bifolia & c & . & . & + & \\
\hline Rubus sp. & & . & + & . & \\
\hline Peucedanum sp. & & . & + & & \\
\hline
\end{tabular}

\section{Legend:}

a1 - High tree layer,

a2 - Middle tree layer,

$\mathrm{b}$ - Shrub layer,

c - Herb layer.

Coordinates of the relevés:

1 45.8291705 15.5567301; $\quad 245.748087$ 15.639837;

345.7476 15.639782;
445.74766815 .639245$.

Table 5 (Tabela 5): Ass. Vicio oroboidi-Fagetum.

\begin{tabular}{lccccccccccccccccccc} 
Relevé No. & 1 & 2 & 3 & 4 & 5 & 6 & 7 & 8 & 9 & 10 & 11 & 12 & 13 & 14 & 15 & 16 & 17 & 18 & 19 \\
Date 2010. (Day/Month) & $9 / 6$ & $9 / 6$ & $9 / 6$ & $26 / 5$ & $26 / 5$ & $26 / 5$ & $26 / 5$ & $26 / 5$ & $26 / 5$ & $21 / 5$ & $21 / 5$ & $21 / 5$ & $21 / 5$ & $29 / 6$ & $29 / 6$ & $29 / 6$ & $29 / 6$ & $29 / 6$ & $29 / 6$ \\
Elevation (m) & 350 & 557 & 733 & 568 & 707 & 515 & 558 & 714 & 716 & 675 & 700 & 687 & 774 & 238 & 593 & 223 & 643 & 648 & 870 \\
Aspect $\left(^{\circ}\right)$ & 40 & 10 & 360 & 315 & 225 & 60 & 45 & 110 & 75 & 30 & 260 & 45 & 330 & 33 & 315 & 360 & 30 & 75 & 130 \\
Slope $\left({ }^{\circ}\right)$ & 50 & 35 & 35 & 10 & 25 & 10 & 10 & 15 & 5 & 30 & 40 & 50 & 5 & 30 & 5 & 40 & 30 & 30 & 5 \\
Cover of layers (\%): & & & & & & & & & & & & & & & & & & \\
Tree (high) & 100 & 100 & 100 & 100 & 90 & 100 & 90 & 100 & 90 & 100 & 100 & 100 & 90 & 100 & 100 & 100 & 90 & 100 & 100 \\
Tree (middle) & 5 & 10 & 30 & 50 & 20 & 20 & 30 & 5 & 20 & 15 & 10 & 10 & 5 & 20 & 10 & 5 & 5 & 3 & 10 \\
Shrub & 50 & 30 & 5 & 15 & 10 & 3 & 2 & 25 & 20 & 5 & 5 & 40 & 10 & 5 & 20 & 3 & 5 & 30 & 5 \\
Herb & 50 & 30 & 50 & 10 & 70 & 30 & 20 & 65 & 70 & 60 & 80 & 80 & 90 & 30 & 80 & 30 & 60 & 70 & 80 \\
\hline
\end{tabular}

Charact. species of the ass.

Epimedium alpinum

Vicia oroboides

Epimedio-Fagenion

Aposeris foetida

Hacquetia epipactis

Ruscus hypoglossum

Aremonio-Fagion

Staphylea pinnata

Daphne laureola

Cyclamen purpurascens

Lamium orvala

Omphalodes verna

Aremonia agrimonoides

Cardamine kitaibelii

Euphorbia carniolica

Helleborus niger

Cardamine trifolia 


\section{Relevé No.}

\section{Fagetalia}

Fagus sylvatica

Fagus sylvatica

Fagus sylvatica

Fagus sylvatica

Daphne mezereum

Crataegus laevigata

Galium odoratum

Cardamine bulbifera

Sanicula europaea

Pulmonaria officinalis

Carex sylvatica

Athyrium filix-femina

Dryopteris filix-mas

Lamium galeobdolon

Prenanthes purpurea

Polygonatum multiflorum

Actaea spicata

Viola reichenbachiana

Euphorbia dulcis

Euphorbia amygdaloides

Mercurialis perennis

Asarum europaeum

Lilium martagon

Neottia nidus-avis

Mycelis muralis

Lathyrus vernus

Scrophularia nodosa

Paris quadrifolia

Primula vulgaris

Ranunculus lanuginosus

Brachypodium sylvaticum

Allium ursinum

Leucojum vernum

Epilobium montanum

Festuca altissima

\section{Carpino-Fagetea}

Quercus petraea

Quercus petraea

Acer pseudoplatanus

Acer pseudoplatanus

Acer pseudoplatanus

Carpinus betulus

Carpinus betulus

Ulmus glabra

Ulmus glabra

Prunus avium

Prunus avium

Acer campestre

Rosa arvensis

Lonicera xylosteum

$$
\begin{aligned}
& \begin{array}{llllllllllllllllllll}
\text { a1 } & 5 & 5 & 4 & 5 & 5 & 4 & 4 & 5 & 5 & 5 & 5 & 5 & 5 & 5 & 5 & 5 & 5 & 5 & 5
\end{array} \\
& \mathrm{a} 2+\begin{array}{lllllllllllllllll} 
& 1 & 2 & 3 & 2 & 2 & 1 & 1 & 2 & 2 & 1 & 1 & 1 & 2 & 1 & 1 & 1 \\
+ & 1
\end{array}
\end{aligned}
$$

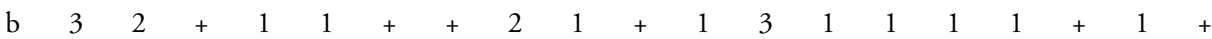

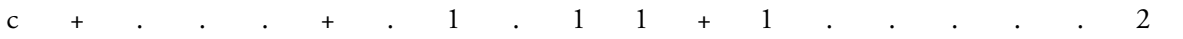

$$
\begin{aligned}
& \text { b }
\end{aligned}
$$

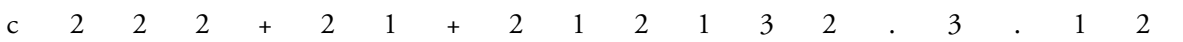

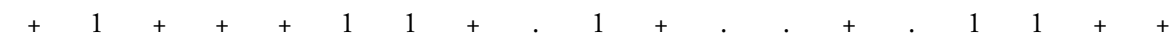

$$
\begin{aligned}
& \begin{array}{llllllllllllllllll}
\cdot & + & + & + & \cdot & \cdot & & + & + & + & 1 & + & + & 1 & + & + & + & + \\
+ & \cdot & + & + & + & . & . & + & + & + & + & + & + & + & + & + & +
\end{array}
\end{aligned}
$$

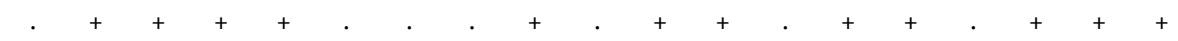

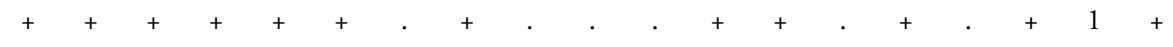

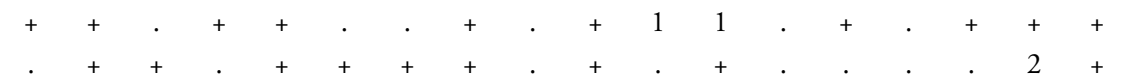

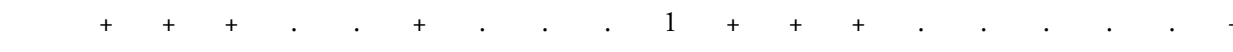

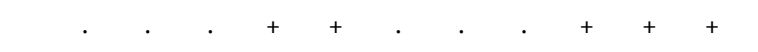

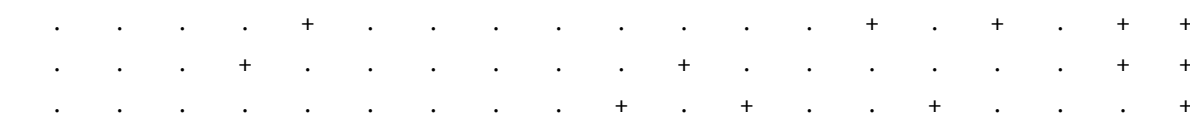

$$
\begin{aligned}
& \text { (1) }
\end{aligned}
$$

s


Ulmus glabra

Tilia platyphyllos

Populus tremula

Acer platanoides

Anemone nemorosa

Hedera helix

Symphytum tuberosum

Melica uniflora

Galium sylvaticum

Luzula pilosa

Polystichum aculeatum

Carex pilosa

Hieracium murorum

Glechoma hirsuta

Poa nemoralis

Polygonatum verticillatum

Convallaria majalis

Crocus purpureus

Arum maculatum

Carex digitata

Cephalanthera damasonium

Polystichum setiferum

Stellaria holostea

Galium schultesii

Lathyrus niger

\section{Quercetea roboris}

Castanea sativa

Castanea sativa

Castanea sativa

Betula pendula

Luzula luzuloides

Melampyrum pratense

\section{Vaccinio-Piceetea}

Picea abies

Oxalis acetosella

Maianthemum bifolium

Quercetea pubescentis

Quercus cerris

Ostrya carpinifolia

Sorbus torminalis

Tamus communis

Cephalanthera longifolia

Festuca heterophylla

Hieracium racemosum

Rhamno-Prunetea

Rubus hirtus

Corylus avellana

Crataegus monogyna

\section{Robinietea}

Clematis vitalba

Robinia pseudoacacia

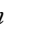


Relevé No.

Sambucus nigra

Epilobietea angustifolii

Salvia glutinosa

Aegopodium podagraria

Senecio ovatus

\section{Trifolio-Geranietea}

Potentilla micrantha

Solidago virgaurea

Galium mollugo

\section{Mulgedio-Aconitetea}

Doronicum austriacum

Phyteuma ovatum

Festuco-Brometea

Veronica austriaca

Cruciata glabra

Carex flacca

Dorycnium germanicum

Molinio-Arrhenatheretea

Gentiana asclepiadea

Platanthera bifolia

Ajuga reptans

Asplenietea trichomanis

Polypodium vulgare

Asplenium scolopendrium

Other species

Pteridium aquilinum

Poa sp.

Legend: a1 - High tree layer, a2 - Middle tree layer, b - Shrub layer, c - Herb layer.

\section{Coordinates of the relevés:}

1 45.7325168 15.5860148; 245.773606 15.6202815; 3 45.8126617 15.544225; 4 45.714703 15.338975;

5 45.725445 15.370827; 6 45.7294106 15.4066316; 7 45.7281809 15.4078134; 8 45.7243258 15.369575;

$945.725187515 .3693379 ; 1045.8194708$ 15.5196302; 11 45.8173445 15.5152848; 1245.8170271 15.5154358;

1345.7968954 15.4954816; 1445.6719983 15.4672067; 15 45.7461280 15.678812; 1645.6715753 15.468671;

$1745.7155415 .3850633 ; 1845.719489715 .3854567 ; 1945.778595815 .3912386$. 
Table 6 (Tabela 6): Ass. Hacquetio-Fagetum.

\begin{tabular}{|c|c|c|c|c|c|c|c|c|c|c|c|c|c|c|}
\hline Relevé No. & 1 & 2 & 3 & 4 & 5 & 6 & 7 & 8 & 9 & 10 & 11 & 12 & 13 & 14 \\
\hline Date 2010 (Day/Month) & $9 / 6$ & $9 / 6$ & $9 / 6$ & $9 / 6$ & $9 / 6$ & $26 / 5$ & $26 / 5$ & $26 / 5$ & $26 / 5$ & $26 / 5$ & $21 / 5$ & $21 / 5$ & $21 / 5$ & $21 / 5$ \\
\hline Elevation $(\mathrm{m})$ & 369 & 353 & 340 & 730 & 327 & 289 & 520 & 525 & 725 & 669 & 533 & 555 & 650 & 510 \\
\hline Aspect $\left({ }^{\circ}\right)$ & 65 & 270 & 225 & 225 & 135 & 135 & 135 & 90 & 270 & 155 & 275 & 120 & 100 & 10 \\
\hline Slope $\left(^{\circ}\right)$ & 70 & 40 & 40 & 20 & 10 & 40 & 15 & 20 & 00 & 70 & 30 & 40 & 15 & 5 \\
\hline \multicolumn{15}{|l|}{ Cover of layers (\%): } \\
\hline Tree (high) & 90 & 100 & 100 & 90 & 90 & 90 & 90 & 80 & 90 & 90 & 80 & 80 & 100 & 90 \\
\hline Tree (middle) & 3 & 20 & 40 & 30 & 40 & 30 & 40 & 50 & 50 & 5 & 10 & 20 & 10 & 20 \\
\hline Shrub & 60 & 40 & 40 & 10 & 30 & 40 & 20 & 50 & 10 & 10 & 30 & 30 & 10 & 20 \\
\hline Herb & 40 & 90 & 50 & 50 & 40 & 90 & 50 & 60 & 70 & 70 & 90 & 30 & 90 & 70 \\
\hline Subass. & \multicolumn{14}{|c|}{ typicum } \\
\hline
\end{tabular}

Charact. species of the ass.

Hacquetia epipactis

Aposeris foetida

Primula vulgaris

Asarum europaeum

Cardamine enneaphyllos

Diff. species of the subass.

Acer obtusatum

Acer obtusatum

Acer obtusatum

Vincetoxicum hirundinaria

Epimedio-Fagenion

Epimedium alpinum

Knautia drymeia

Helleborus odorus

Ruscus hypoglossum

Vicia oroboides

Aremonio-Fagion

Staphylea pinnata

Lonicera caprifolium

Cyclamen purpurascens

Aremonia agrimonoides

Lamium orvala

Omphalodes verna

Helleborus niger

Euphorbia carniolica

Homogyne sylvestris

Helleborus dumetorum

Cardamine trifolia

Calamintha grandiflora

\section{Fagetalia}

Fagus sylvatica

Fagus sylvatica

Fagus sylvatica

c

(1)

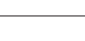




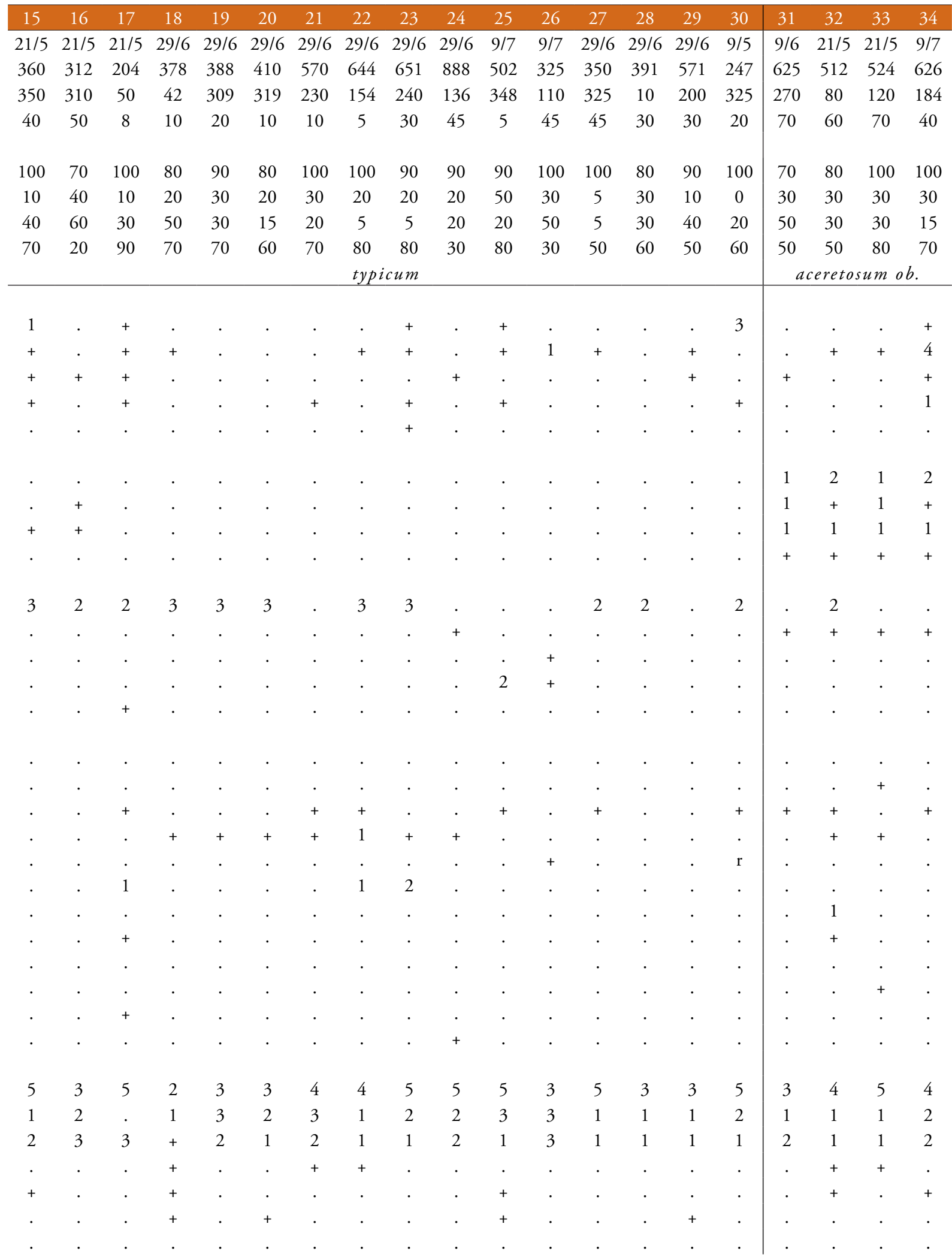




$\begin{array}{lllllllllllllll}\text { Relevé No. } & 1 & 2 & 3 & 4 & 5 & 6 & 7 & 8 & 9 & 10 & 11 & 12 & 13 & 14\end{array}$

Pulmonaria officinalis

Sanicula europaea

Galium odoratum

Cardamine bulbifera

Lathyrus vernus

Euphorbia dulcis

Dryopteris filix-mas

Neottia nidus-avis

Carex sylvatica

Brachypodium sylvaticum

Viola reichenbachiana

Lilium martagon

Prenanthes purpurea

Mycelis muralis

Lamium galeobdolon

Mercurialis perennis

Polygonatum multiflorum

Actaea spicata

Phyteuma spicatum

Campanula trachelium

Festuca altissima

Euphorbia amygdaloides

Paris quadrifolia

Circaea lutetiana

Athyrium filix-femina

\section{Carpino-Fagetea}

Quercus petraea

Quercus petraea

Quercus petraea

Quercus petraea

Carpinus betulus

c

$\begin{array}{llllllllllllll}+ & + & + & . & + & + & + & + & . & + & + & + & + & + \\ + & + & + & 1 & + & + & + & . & . & . & 1 & + & . \\ 2 & . & 1 & 1 & 2 & . & . & + & . & + & . & 1 & 3\end{array}$

Carpinus betulus

Carpinus betulus

Acer pseudoplatanus

Acer pseudoplatanus

Acer pseudoplatanus

Acer campestre

Acer campestre

Prunus avium

Prunus avium

Populus tremula

Populus tremula

Acer platanoides

Acer platanoides

Ulmus glabra

Ulmus glabra

Tilia platyphyllos

Tilia platyphyllos

Pyrus pyraster

Pyrus pyraster

a1

a2

b

a1

a2

b

a2

b

a1

a1

a1

b 


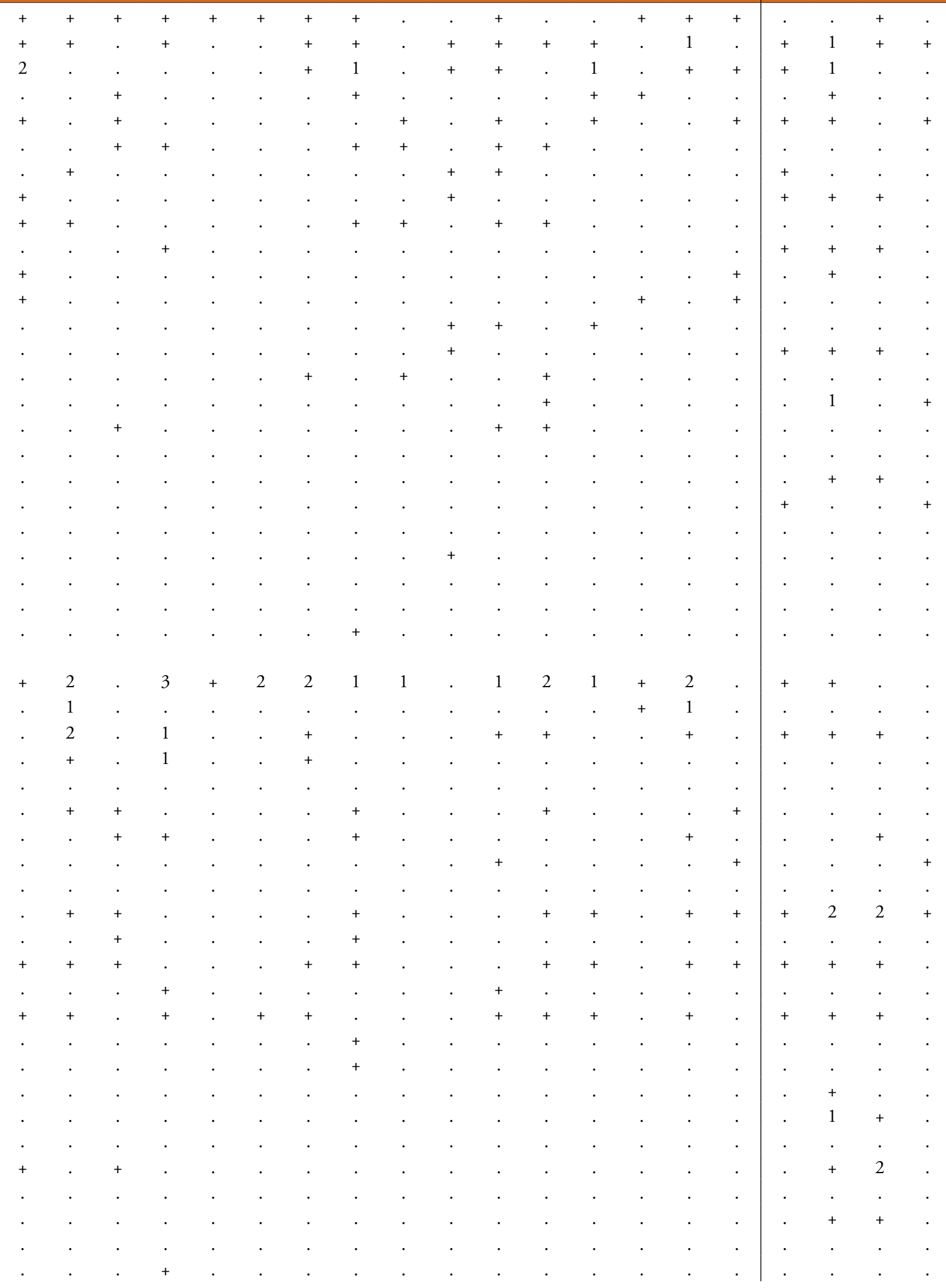




\begin{tabular}{lllllllllllllll}
\hline Relevé No. & 1 & 2 & 3 & 4 & 5 & 6 & 7 & 8 & 9 & 10 & 11 & 12 & 13 & 14
\end{tabular}

Rosa arvensis

Ilex aquifolium

Lonicera xylosteum

Euonymus latifolius

Euonymus europaeus

Abies alba

Hedera helix

Galium sylvaticum

Melittis melissophyllum

Carex digitata

Hieracium murorum

Anemone nemorosa

Convallaria majalis

Cephalanthera damasonium

Carex pilosa

Symphytum tuberosum

Melica uniflora

Luzula pilosa

Melica nutans

Lathyrus niger

Aruncus dioicus

Hepatica nobilis

Galium schultesii

Polystichum aculeatum

Poa nemoralis

Aconitum lycoctonum

Glechoma hirsuta

Cardamine impatiens

Stellaria holostea

Milium effusum

Cephalanthera rubra

Quercetea pubescentis

Quercus cerris

Quercus cerris

Quercus cerris

Sorbus torminalis

Sorbus torminalis

Sorbus torminalis

Sorbus aria

Sorbus aria

Fraxinus ornus

Fraxinus ornus

Ostrya carpinifolia

Tamus communis

Viburnum lantana

Cornus mas

Genista tinctoria

Sorbus domestica

Cephalanthera longifolia

Festuca heterophylla

$\begin{array}{lllll}\mathrm{a} 1 & \cdot & + & \cdot & + \\ \mathrm{a} 2 & \cdot & \cdot & \cdot & \cdot \\ \mathrm{b} & \cdot & \cdot & \cdot & \cdot \\ \mathrm{a} 1 & \cdot & + & \cdot & \cdot \\ \mathrm{a} 2 & \cdot & \cdot & \cdot & \cdot \\ \mathrm{b} & \cdot & \cdot & \cdot & \cdot \\ \mathrm{a} 2 & \cdot & + & \cdot & \cdot \\ \mathrm{b} & \cdot & + & \cdot & \cdot \\ \mathrm{a} 2 & \cdot & \cdot & \cdot & \cdot \\ \mathrm{b} & + & + & \cdot & \cdot \\ \mathrm{a} 1 & \cdot & \cdot & \cdot & \cdot \\ \mathrm{b} & \cdot & + & + & + \\ & \cdot & + & + & \cdot \\ & + & + & 1 & \cdot \\ & \cdot & \cdot & \cdot & \cdot \\ \mathrm{c} & + & \cdot & + & + \\ & \cdot & \cdot & +\end{array}$




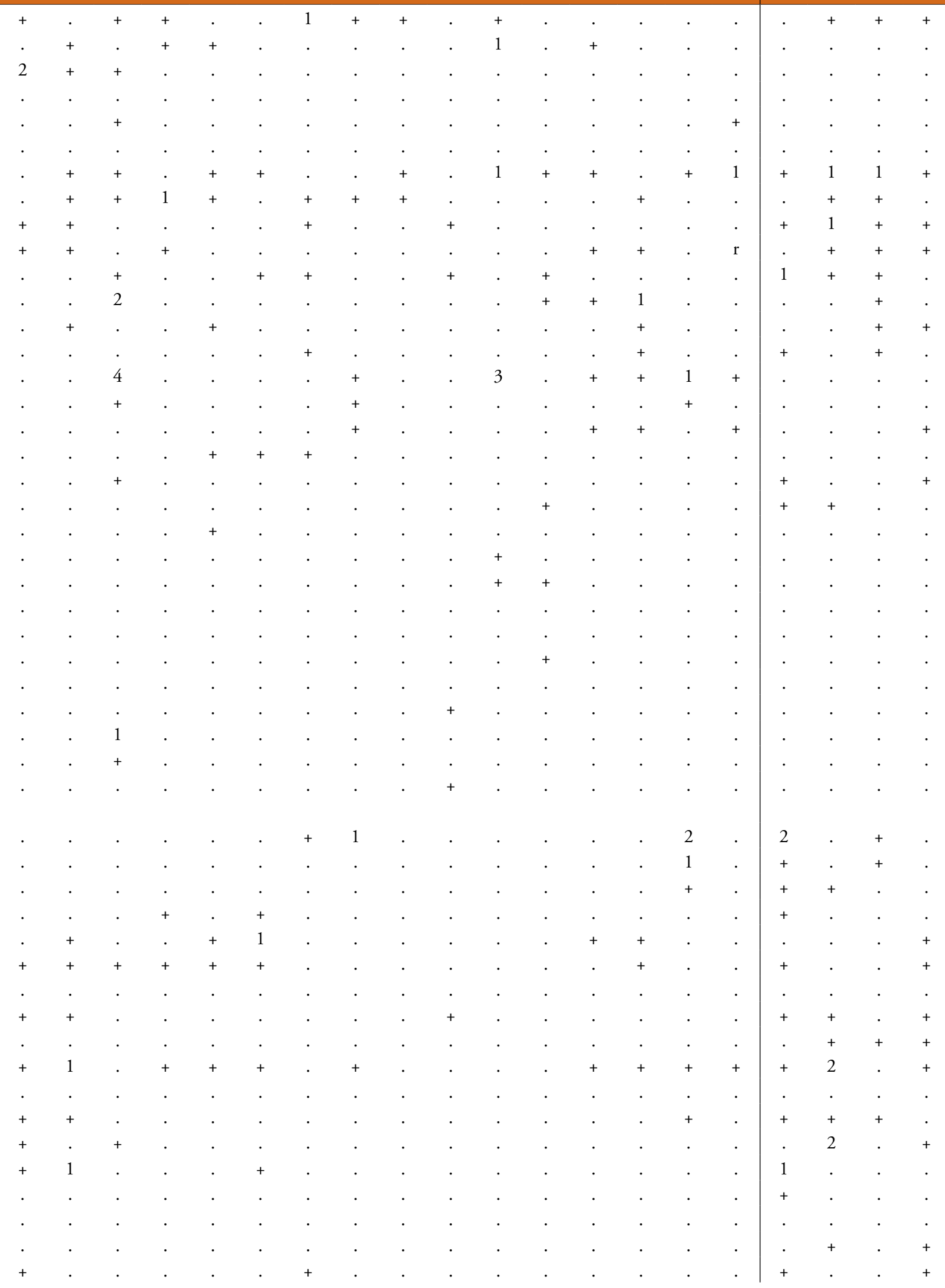


Hieracium racemosum

Clinopodium vulgare

Aristolochia lutea

Mercurialis ovata

Lathyrus venetus

\section{Quercetea roboris}

Castanea sativa

Castanea sativa

Castanea sativa

Castanea sativa

Betula pendula

Serratula tinctoria

Luzula luzuloides

Melampyrum pratense

Luzula forsteri

\section{Vaccinio-Piceetea}

Picea abies

Pyrola rotundifolia

Oxalis acetosella

\section{Erico-Pinetea}

Carex alba

\section{Rhamno-Prunetea}

Rubus hirtus

Cornus sanguinea

Crataegus monogyna

Corylus avellana

Berberis vulgaris

Juniperus communis

Viburnum opulus

Ligustrum vulgare

Rhamnus cathartica

Prunus spinosa

\section{Robinietea}

Clematis vitalba

Sambucus nigra

\section{Epilobietea angustifolii}

Salvia glutinosa

Fragaria vesca

Aegopodium podagraria

Heracleum sphondylium

Digitalis grandiflora

Geranium phaeum

Senecio ovatus

Betonica officinalis

Valeriana officinalis

\section{Trifolio-Geranietea}

Chamaecytisus hirsutus

Tanacetum corymbosum 
1.2

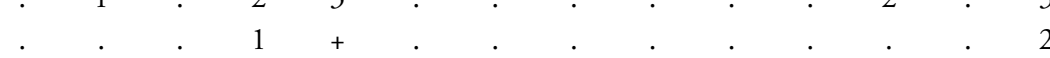

2

3
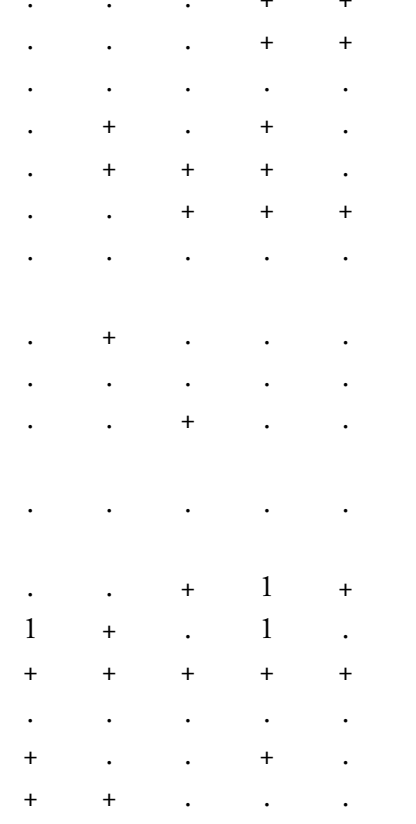

1
+
+ 


\begin{tabular}{|c|c|c|c|c|c|c|c|c|c|c|c|c|c|c|c|}
\hline Relevé No. & & 1 & 2 & 3 & 4 & 5 & 6 & 7 & 8 & 9 & 10 & 11 & 12 & 13 & 14 \\
\hline Campanula persicifolia & & . & . & . & . & . & . & . & . & + & . & + & . & + & . \\
\hline Veronica chamaedrys & & . & . & . & . & . & . & . & . & . & . & . & + & . & . \\
\hline Iris graminea & & . & . & . & . & . & . & . & . & . & . & . & . & . & . \\
\hline Silene nutans & & . & . & . & . & . & . & . & . & . & . & . & . & . & . \\
\hline Laser trilobum & & . & . & . & . & . & . & . & . & . & . & . & . & . & . \\
\hline Hypericum montanum & & . & . & . & . & . & . & . & . & . & . & . & . & . & . \\
\hline \multicolumn{16}{|l|}{ Mulgedio-Aconitetea } \\
\hline Phyteuma ovatum & c & + & + & + & . & + & + & . & . & + & + & . & . & . & . \\
\hline Thalictrum aquilegiifolium & & . & . & . & . & . & . & . & . & . & . & + & . & . & . \\
\hline Doronicum austriacum & & . & + & . & . & . & . & . & + & . & . & . & . & . & . \\
\hline Cirsium erisithales & & . & . & . & . & . & . & . & . & + & . & . & . & . & . \\
\hline \multicolumn{16}{|l|}{ Festuco-Brometea } \\
\hline Cruciata glabra & c & + & . & . & . & + & . & . & . & . & + & + & + & + & 1 \\
\hline Carex flacca & & . & . & . & . & . & . & . & . & . & + & + & . & + & . \\
\hline Anthericum ramosum & & . & . & . & . & . & . & . & . & . & . & 2 & . & . & . \\
\hline Arabis hirsuta & & . & . & . & . & . & . & . & . & . & . & . & . & . & . \\
\hline \multicolumn{16}{|l|}{ Molinio-Arrbenatheretea } \\
\hline Gentiana asclepiadea & c & + & + & + & + & + & + & + & . & . & + & + & + & . & . \\
\hline Platanthera bifolia & & . & + & . & + & + & . & + & + & + & . & + & . & + & . \\
\hline Ajuga reptans & & + & . & . & + & . & . & . & . & . & . & . & . & . & . \\
\hline Dactylis glomerata & & . & . & . & . & . & . & . & . & . & . & . & . & . & . \\
\hline Taraxacum officinale & & . & . & . & . & . & . & . & . & . & . & . & . & $\mathrm{r}$ & . \\
\hline \multicolumn{16}{|l|}{ Asplenietea trichomanis } \\
\hline Polypodium vulgare & c & + & + & . & . & + & . & . & . & . & . & . & . & . & . \\
\hline Asplenium scolopendrium & & . & . & . & . & . & . & . & 1 & . & . & . & . & . & . \\
\hline Asplenium trichomanes & & . & . & . & . & . & . & . & + & . & + & . & . & . & . \\
\hline \multicolumn{16}{|l|}{ Other species } \\
\hline Malus sylvestris & $\mathrm{b}$ & . & . & . & . & . & . & . & . & . & . & . & . & . & . \\
\hline Frangula alnus & & . & . & . & . & . & . & . & . & . & . & . & . & . & . \\
\hline Pteridium aquilinum & c & + & + & + & + & + & . & + & + & + & + & + & . & + & + \\
\hline Rubus sp. & & . & . & . & . & . & . & . & . & . & . & . & . & . & . \\
\hline Orchis sp. & & . & . & . & . & . & . & . & . & . & . & . & . & . & \\
\hline
\end{tabular}

Legend: a1 - High tree layer, a2 - Middle tree layer, b - Shrub layer, c - Herb layer.

\section{Coordinates of the relevés:}

1 45.73193 15.58546; 2 45.7324883 15.586955; 3 45.7344283 15.5856017; 4 45.7652483 15.5999733;

5 45.781455 15.6404417; 645.705087 15.456413; 7 45.728812 15.412297; 8 45.729803 15.404405; 9 45.765137 15.367078;

1045.7591014 15.3709664; 1145.8290343 15.5563338; 1245.8256753 15.5533546; 1345.7657176 15.4506171;

14 45.7467283 15.4665097; 15 45.7292171 15.4570139; 16 45.7234695 15.4592164; 1745.7031623 15.4865234;

$1845.6748415 .4409367 ; 1945.6714215 .4376217 ; 2045.6677617$ 15.4311183; 2145.71184 15.388115;

$2245.71494515 .385295 ; 23$ 45.7194917 15.3842883; 24 45.767287 15.38527; 25 45.74379 15.66779;

$2645.75931315 .661213 ; 2745.6725002$ 15.4374473; 28 45.6689466 15.4333993; 2945.7121667 15.3863054;

3045.6762659 15.3936626; 31 45.7572755 15.565091; 3245.8355808 15.5656181; 3345.831667 15.5599682;

3445.74406715 .647915$. 


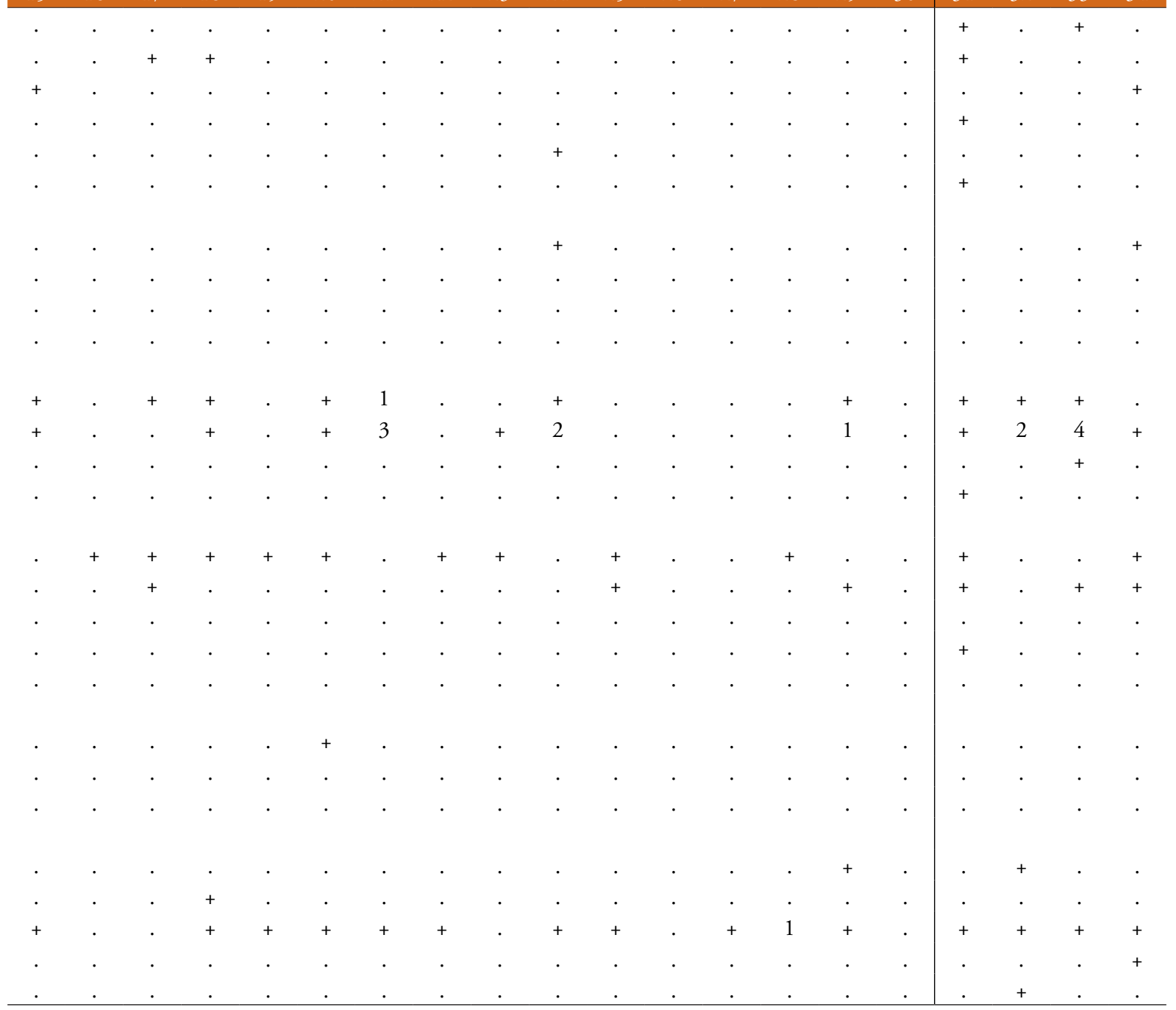


Table 7 (Tabela 7): Lamio orvalae-Fagetum.

\begin{tabular}{|c|c|c|c|c|c|c|c|c|c|c|c|c|c|c|c|c|c|c|c|}
\hline Relevé No. & 1 & 2 & 3 & 4 & 5 & 6 & 7 & 8 & 9 & 10 & 11 & 12 & 13 & 14 & 15 & 16 & 17 & 18 & 19 \\
\hline Date 2010 (Day/Month) & $9 / 6$ & $9 / 6$ & $9 / 6$ & $9 / 6$ & $9 / 6$ & $9 / 6$ & $9 / 6$ & $9 / 6$ & $9 / 6$ & $9 / 6$ & $9 / 6$ & $9 / 6$ & $26 / 5$ & $26 / 5$ & $26 / 5$ & $26 / 5$ & $26 / 5$ & $26 / 5$ & $21 / 5$ \\
\hline Elevation $(\mathrm{m})$ & 530 & 702 & 789 & 464 & 613 & 664 & 727 & 304 & 518 & 791 & 484 & 660 & 688 & 851 & 273 & 491 & 874 & 575 & 759 \\
\hline Aspect $\left({ }^{\circ}\right)$ & 250 & 360 & 290 & 80 & 230 & 30 & 60 & 80 & 225 & 360 & 45 & 225 & 45 & 45 & 70 & 40 & 45 & 330 & 360 \\
\hline Slope $\left(^{\circ}\right)$ & 50 & 35 & 70 & 45 & 40 & 40 & 30 & 15 & 40 & 30 & 35 & 40 & 45 & 35 & 40 & 60 & 20 & 25 & 15 \\
\hline \multicolumn{20}{|l|}{ Cover of layers $(\%)$ : } \\
\hline Tree (high) & 100 & 90 & 100 & 100 & 100 & 100 & 100 & 80 & 100 & 100 & 100 & 100 & 100 & 90 & 100 & 100 & 100 & 100 & 80 \\
\hline Tree (middle) & 10 & 5 & 5 & 20 & 20 & 5 & 15 & 30 & 60 & 20 & 20 & 30 & 20 & 20 & 10 & 10 & 5 & 10 & 30 \\
\hline Shrub & 5 & 5 & 30 & 3 & 5 & 20 & 20 & 40 & 5 & 15 & 10 & 30 & 10 & 10 & 5 & 5 & 50 & 5 & 30 \\
\hline Herb & 60 & 40 & 70 & 20 & 60 & 90 & 50 & 70 & 50 & 80 & 50 & 70 & 8 & 80 & 60 & 50 & 90 & 80 & 80 \\
\hline Subass. & \multicolumn{19}{|c|}{ typicum } \\
\hline
\end{tabular}

Charact. species of the ass.

Lamium orvala

Diff. species of the subass.

Fraxinus ornus

Fraxinus ornus

Acer obtusatum

Acer obtusatum

Acer obtusatum

Cornus sanguinea

Carex flacca

Lamio orvalae-Fagenion

Cardamine kitaibelii

Cardamine trifolia

Scopolia carniolica

Aremonio-Fagion

Staphylea pinnata

Lonicera caprifolium

Cyclamen purpurascens

Aposeris foetida

Hacquetia epipactis

Helleborus odorus

Ruscus hypoglossum

Helleborus niger

Aremonia agrimonoides

Cardamine enneaphyllos

Vicia oroboides

Knautia drymeia

Homogyne sylvestris

Euphorbia carniolica

Epimedium alpinum

Omphalodes verna

Isopyrum thalictroides

Fagetalia

Fagus sylvatica

Fagus sylvatica

Fagus sylvatica

Fagus sylvatica

Tilia cordata

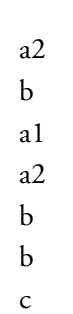

Tilia cordata

$\begin{array}{lllllllllllllllllllll}\mathrm{a} 1 & 5 & 5 & 5 & 5 & 4 & 5 & 5 & 4 & 3 & 5 & 4 & 5 & 5 & 5 & 5 & 5 & 5 & 4 & 4 \\ \mathrm{a} 2 & 2 & 1 & 1 & 2 & 2 & 1 & 1 & 2 & 3 & 1 & 1 & 3 & 2 & 2 & + & 1 & + & 1 & 2 \\ \mathrm{~b} & + & + & 1 & + & + & 2 & + & 2 & + & . & + & + & . & 1 & 1 & 1 & 1 & 1 & 2 \\ \mathrm{c} & . & . & + & + & . & 1 & . & . & . & + & . & . & + & . & + & + & . & . & + \\ \mathrm{a} 1 & . & . & . & . & . & . & . & . & . & . & . & . & . & . & . & . & . & . & . \\ \mathrm{b} & . & . & . & . & . & . & . & . & . & . & . & . & . & . & . & . & . & . & .\end{array}$


\begin{tabular}{lllllllllll|llllllllllllllll}
20 & 21 & 22 & 23 & 24 & 25 & 26 & 27 & 28 & 29 & 30 & 31 & 32 & 33 & 34 & 35 & 36 & 37 & 38 & 39 & 40 & 41 & 42 & 43 & 44 & 45
\end{tabular} $\begin{array}{llllllllllllllllllllllllllll}21 / 5 & 9 / 7 & 9 / 7 & 9 / 7 & 9 / 7 & 9 / 7 & 9 / 7 & 9 / 7 & 9 / 7 & 9 / 7 & 29 / 6 & 9 / 6 & 9 / 6 & 26 / 5 & 26 / 5 & 26 / 5 & 26 / 5 & 21 / 5 & 29 / 6 & 9 / 7 & 9 / 7 & 9 / 7 & 9 / 7 & 9 / 7 & 9 / 7 & 9 / 7\end{array}$

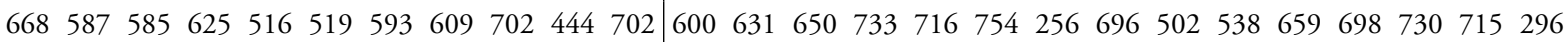
\begin{tabular}{lllllllllll|lllllllllllllll}
55 & 210 & 354 & 78 & 246 & 345 & 307 & 78 & 170 & 23 & 80 & 225 & 225 & 225 & 110 & 280 & 125 & 120 & 145 & 177 & 153 & 112 & 234 & 309 & 59 & 247
\end{tabular} \begin{tabular}{lllllllllll|lllllllllllllll}
40 & 20 & 20 & 30 & 2 & 55 & 25 & 40 & 10 & 35 & 25 & 30 & 15 & 40 & 40 & 35 & 70 & 80 & 10 & 20 & 15 & 5 & 10 & 20 & 40 & 65
\end{tabular}

\begin{tabular}{lllllllllll|lllllllllllllll}
100 & 90 & 100 & 100 & 100 & 100 & 100 & 100 & 100 & 100 & 100 & 100 & 100 & 100 & 80 & 90 & 80 & 100 & 80 & 100 & 90 & 100 & 100 & 80 & 100 & 30
\end{tabular} \begin{tabular}{lllllllllll|lllllllllllllllll}
5 & 15 & 10 & 30 & 20 & 10 & 5 & 20 & 20 & 50 & 5 & 40 & 50 & 20 & 20 & 15 & 20 & 30 & 20 & 15 & 20 & 15 & 20 & 30 & 30 & 30
\end{tabular} \begin{tabular}{lllllllllll|lllllllllllllllllll}
5 & 25 & 10 & 70 & 50 & 30 & 60 & 5 & 20 & 20 & 20 & 50 & 10 & 10 & 20 & 15 & 10 & 30 & 50 & 80 & 70 & 20 & 10 & 20 & 10 & 50
\end{tabular} \begin{tabular}{lllllllllll|lllllllllllllll}
90 & 60 & 50 & 80 & 50 & 40 & 50 & 10 & 80 & 50 & 90 & 60 & 70 & 80 & 70 & 60 & 60 & 70 & 100 & 50 & 50 & 80 & 20 & 80 & 50 & 30
\end{tabular} typicum fraxinetosum orni

\begin{tabular}{lllllllllll|lllllllllllllllll}
5 & 5 & 5 & 5 & 5 & 5 & 5 & 5 & 5 & 5 & 4 & 3 & 5 & 5 & 5 & 5 & 5 & 5 & 3 & 4 & 4 & 4 & 5 & 4 & 5 & 3 \\
1 & 2 & 1 & 1 & 2 & 1 & 1 & 2 & 1 & 3 & + & 2 & 3 & 2 & 2 & 1 & 1 & 2 & 1 & 1 & 1 & 1 & 2 & 2 & 2 & 1 \\
1 & + & + & 1 & + & 1 & 1 & + & + & 2 & 1 & 1 & + & 1 & 1 & 1 & + & 1 & 1 &. &. & 2 & 1 & 2 & 1 & 1
\end{tabular}


Daphne mezereum

Lonicera alpigena

Crataegus laevigata

Cardamine bulbifera

Galium odoratum

Mercurialis perennis

Dryopteris filix-mas

Pulmonaria officinalis

Carex sylvatica

Sanicula europaea

Mycelis muralis

Actaea spicata

Asarum europaeum

Lathyrus vernus

Polygonatum multiflorum

Primula vulgaris

Prenanthes purpurea

Euphorbia dulcis

Brachypodium sylvaticum

Lamium galeobdolon

Lilium martagon

Viola reichenbachiana

Neottia nidus-avis

Euphorbia amygdaloides

Paris quadrifolia

Campanula trachelium

Allium ursinum

Athyrium flix-femina

Ranunculus lanuginosus

Circaea lutetiana

Scrophularia nodosa

Veronica montana

Phyteuma spicatum

Epipactis helleborine

Festuca altissima

\section{Carpino-Fagetea}

Acer pseudoplatanus

Acer pseudoplatanus

Acer pseudoplatanus

Acer pseudoplatanus

Carpinus betulus

Carpinus betulus

Carpinus betulus

Acer campestre

Acer campestre

Acer campestre

Acer platanoides

Acer platanoides

Acer platanoides

Prunus avium

Prunus avium

$\mathrm{b}$
$\mathrm{b}$
$\mathrm{b}$
$\mathrm{c}$

$\begin{array}{rllllllllllllllllll}\mathrm{c} & + & + & + & 1 & 1 & . & 1 & + & + & . & + & + & + & 1 & 1 & + & . & + \\ \quad & 1 & 1 & + & 1 & + & 2 & 1 & . & 2 & 2 & 2 & + & 2 & . & . & . & + & +\end{array}$

$\begin{array}{lllllllllllllllllll}1 & 1 & \cdot & \cdot & \cdot & \cdot & 2 & \cdot & + & 2 & + & + & + & 1 & + & . & 2 & . & + \\ 1 & + & \cdot & + & . & 1 & + & . & + & + & + & + & + & + & + & 1 & + & . & +\end{array}$

.




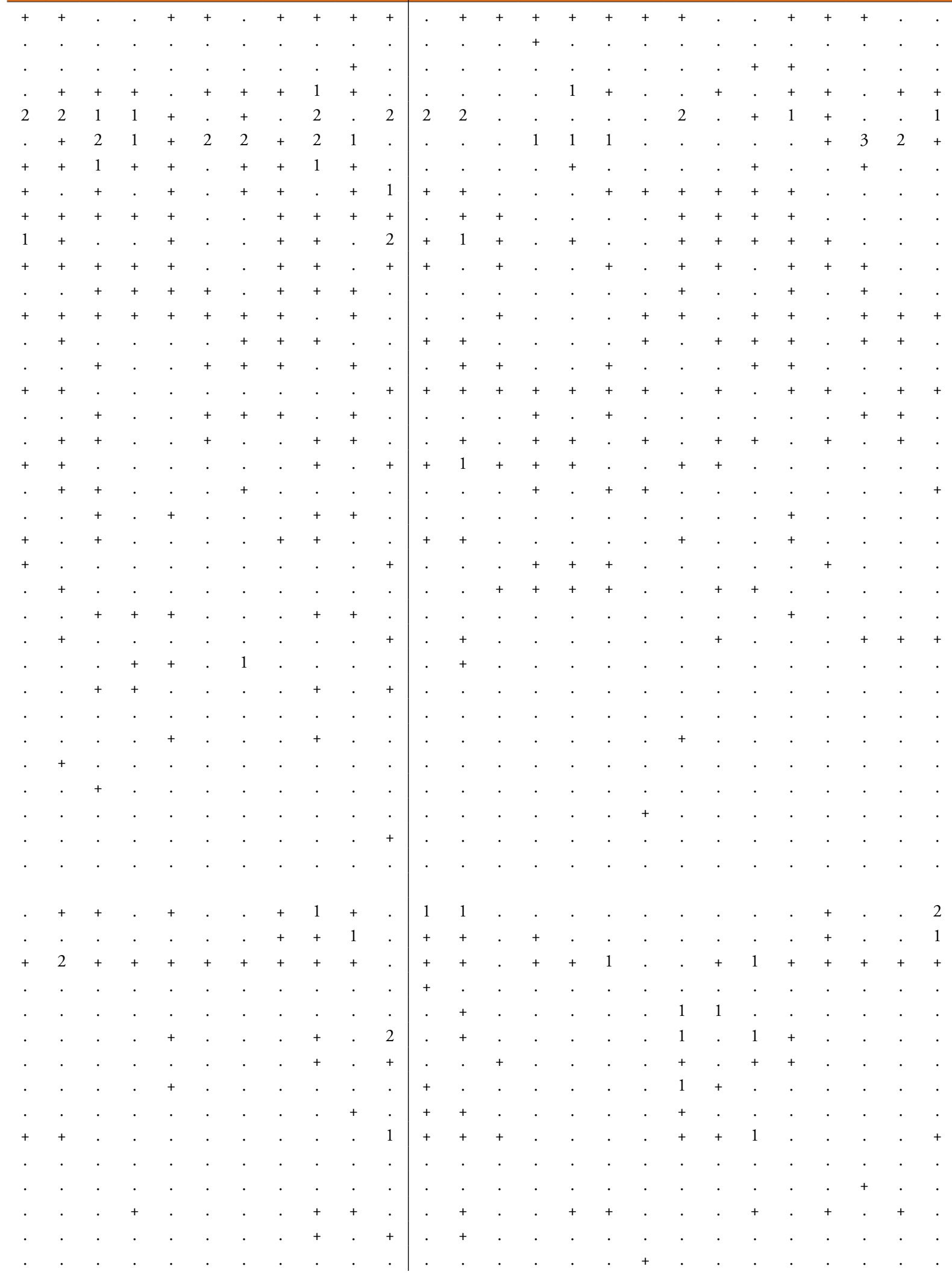


Prunus avium

$\mathrm{b}$

Quercus petraea

Ulmus glabra

Ulmus glabra

Tilia platyphyllos

Tilia platyphyllos

Rosa arvensis

Ilex aquifolium

Euonymus latifolius

Lonicera xylosteum

Tilia cordata

Pyrus pyraster

Euonymus europaeus

Hedera helix

Anemone nemorosa

Carex digitata

Galium sylvaticum

Symphytum tuberosum

Polystichum aculeatum

Cephalanthera damasonium

Aconitum lycoctonum

Arum maculatum

Convallaria majalis

Melica uniflora

Aruncus dioicus

Melica nutans

Glechoma hirsuta

Melittis melissophyllum

Stachys sylvatica

Hepatica nobilis

Galium schultesii

Hieracium murorum

Polystichum setiferum

Carex pilosa

Luzula pilosa

Lathyrus niger

Luzula sylvatica

Stellaria holostea

Moehringia trinervia

Milium effusum

Cerastium sylvaticum

Cephalanthera rubra

Aquilegia vulgaris

Epipactis microphylla

\section{Quercetea pubescentis}

Sorbus aria

Sorbus aria

Sorbus aria

Ostrya carpinifolia

Ostrya carpinifolia

Ostrya carpinifolia 


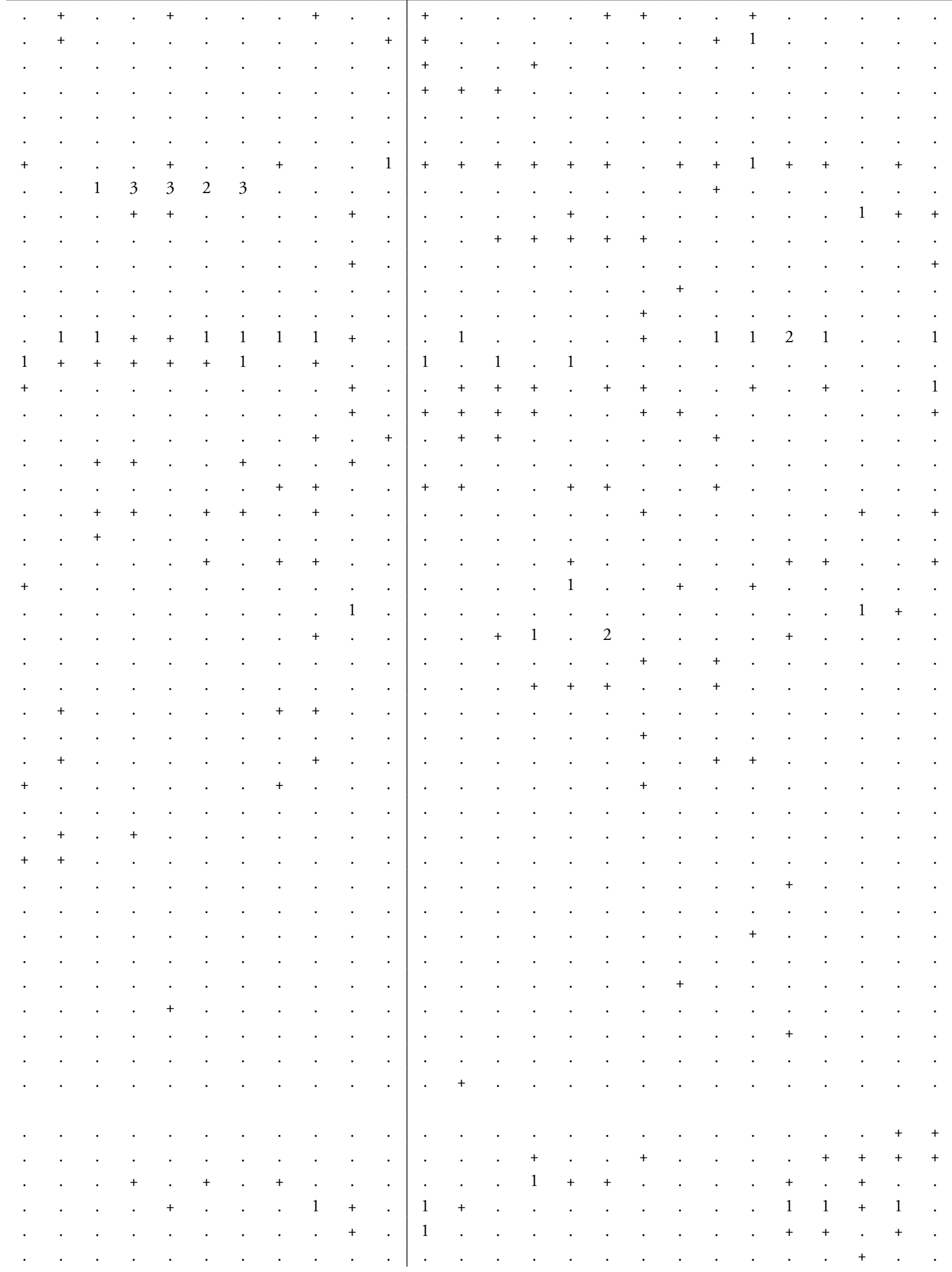




\section{Relevé No.}

$\begin{array}{lllllllllllllllllll}1 & 2 & 3 & 4 & 5 & 6 & 7 & 8 & 9 & 10 & 11 & 12 & 13 & 14 & 15 & 16 & 17 & 18 & 19\end{array}$

Quercus cerris

\section{a1}

Quercus cerris

Sorbus torminalis

Sorbus torminalis

Tamus communis

Viburnum lantana

Vincetoxicum hirundinaria

Cornus mas

Cephalanthera longifolia

Mercurialis ovata

Aristolochia lutea

Lathyrus venetus

Festuca heterophylla

Clinopodium vulgare

Laserpitium latifolium

\section{Quercetea roboris}

Castanea sativa

Castanea sativa

Melampyrum pratense

Luzula luzuloides

Serratula tinctoria

\section{Erico-Pinetea}

Carex alba

Genista januensis

Erica herbacea

Vaccinio-Piceetea

Oxalis acetosella

Maianthemum bifolium

Rhamno-Prunetea

Rubus hirtus

Corylus avellana

Viburnum opulus

Crataegus monogyna

Ligustrum vulgare

Berberis vulgaris

Rhamnus cathartica

Prunus spinosa

Sorbus aucuparia

\section{Robinietea}

Sambucus nigra

Clematis vitalba

Rubus idaeus

Rubus plicatus

\section{Epilobietea angustifolii}

Salvia glutinosa

Heracleum sphondylium

Senecio ovatus

Fragaria vesca

Aegopodium podagraria

Silene dioica

Geranium phaeum b

c

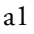

b

c

c

c

b

b 


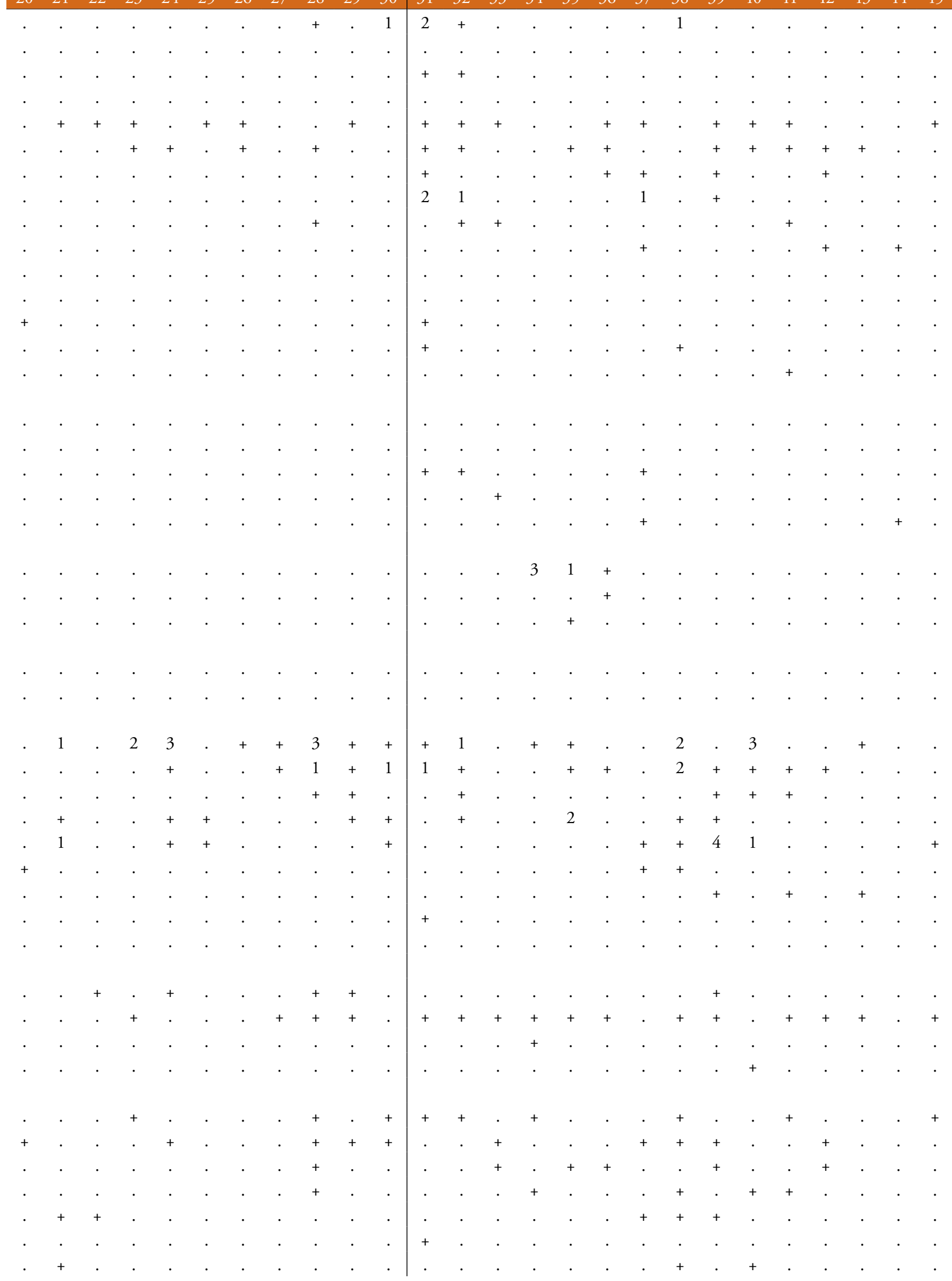


Hypericum hirsutum

Galium lucidum

Impatiens noli-tangere

Glechoma hederacea

Atropa bella-donna

Geranium robertianum

Geum urbanum

Digitalis grandiflora

\section{Trifolio-Geranietea}

Chamaecytisus hirsutus

Tanacetum corymbosum

Solidago virgaurea

Potentilla micrantha

Campanula persicifolia

Iris graminea

Veronica chamaedrys

Tephroseris longifolia

Laser trilobum

Clematis recta

Polygonatum odoratum

Geranium sanguineum

Hypericum montanum

Lathyrus linifolius

Mulgedio-Aconitetea

Phyteuma ovatum

Doronicum austriacum

Lilium carniolicum

Cirsium erisithales

Saxifraga rotundifolia

Festuco-Brometea

Cruciata glabra

Veronica austriaca

Listera ovata

Euphorbia cyparissias

Anthericum ramosum

Molinio-Arrhenatheretea

Astrantia major

Taraxacum officinale

Gentiana asclepiadea

Platanthera bifolia

Asplenietea trichomanis

Asplenium scolopendrium

Asplenium trichomanes

Valeriana tripteris

Polypodium vulgare

\section{Other species}

Juglans regia

Malus sylvestris

Rosa sp.

Pteridium aquilinum

Polystichum lonchitis 


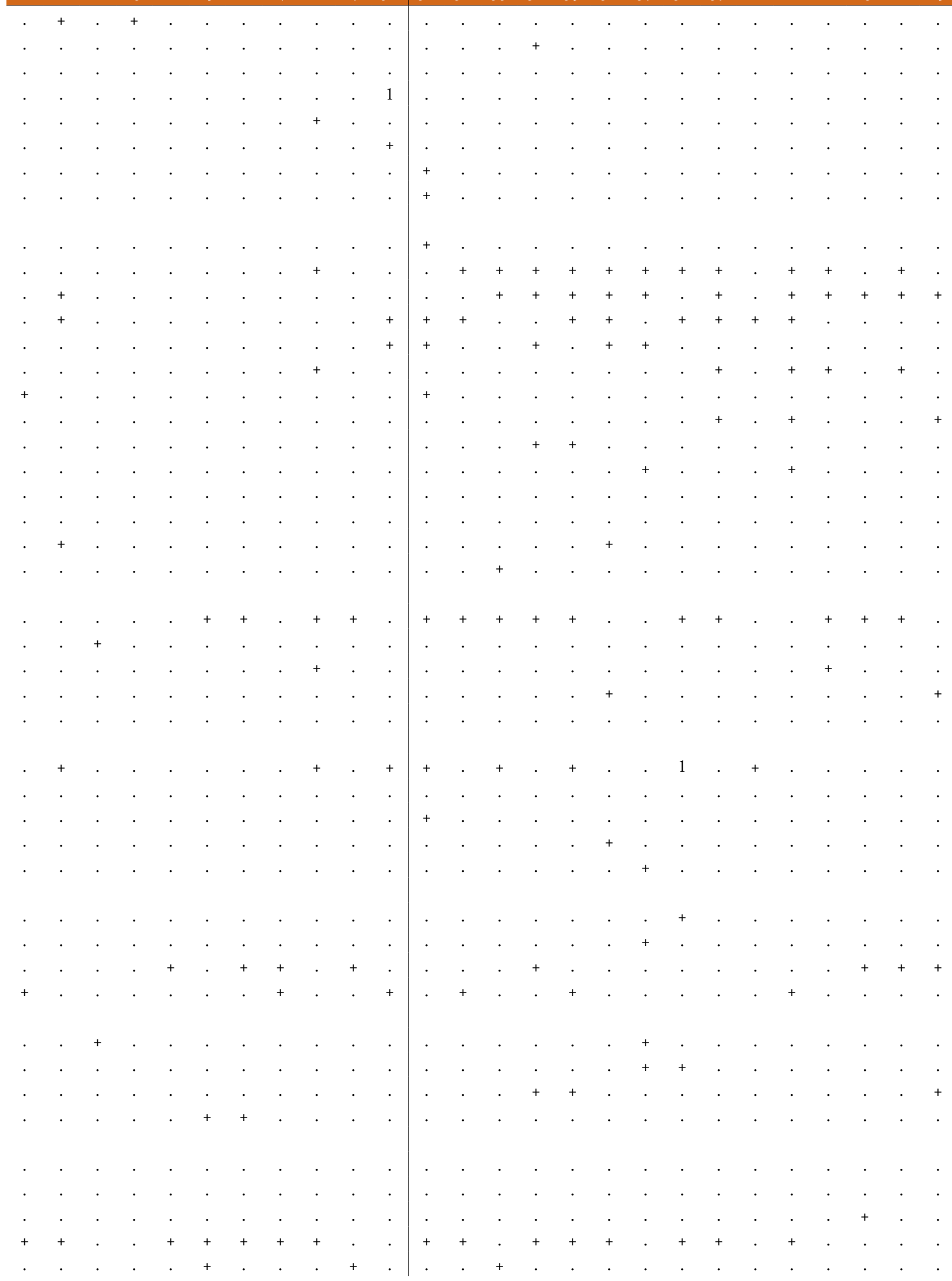




\begin{tabular}{|c|c|c|c|c|c|c|c|c|c|c|c|c|c|c|c|c|c|c|c|}
\hline Relevé No. & 1 & 2 & 3 & 4 & 5 & 6 & 7 & 8 & 9 & 10 & 11 & 12 & 13 & 14 & 15 & 16 & 17 & 18 & 19 \\
\hline Carex pendula & . & . & . & . & . & . & . & . & . & . & . & . & . & . & . & . & . & . & . \\
\hline Sesleria sp. & . & . & . & . & . & . & . & . & . & . & . & . & . & . & . & . & . & . & \\
\hline Festuca sp. & . & . & . & . & . & . & . & . & . & . & . & . & + & . & . & . & . & . & . \\
\hline Orchis sp. & . & . & . & . & . & . & . & . & . & . & . & . & . & . & . & . & . & . & . \\
\hline Arabis sp. & . & . & . & . & . & . & . & . & . & . & . & . & + & . & . & . & . & . & . \\
\hline Peucedanum sp. & . & . & . & . & . & . & . & . & . & . & . & . & . & . & . & . & . & . & . \\
\hline Verbascum sp. & . & . & . & . & . & . & . & . & . & . & . & . & + & . & . & . & . & . & . \\
\hline Rubus sp. & . & . & . & . & . & . & . & . & . & . & . & . & . & . & . & . & . & . & \\
\hline Ornithogalum pyramidale & . & . & . & . & . & . & . & . & . & . & . & . & . & . & . & . & . & . & \\
\hline
\end{tabular}

Legend: a1 - High tree layer, a2 - Middle tree layer, b - Shrub layer, c - Herb layer.

\section{Coordinates of the relevés:}

1 45.7567571 15.5832003; 2 45.7464770 15.5979779; 3 45.7468641 15.6007159; 4 45.7555850 15.5711155; $\mathbf{5} 45.764031815 .5785592 ; 645.7650903$ 15.5849703; 7 45.7632525 15.6002531; 8 45.7808486 15.6411193; $945.757338315 .5819833 ; 1045.748515$ 15.602165; 11 45.7561433 15.5708967; 1245.7658517 15.5891483; 13 45.759662 15.36923; 1445.742907 15.306145; 15 45.7057738 15.456563; 16) 45.7294027 15.4119246; $1745.742523915 .3054183 ; \mathbf{1 8} 45.7151832$ 15.3396807; 1945.8036911 15.4972854; 2045.7663131 15.4499749; $2145.734182015 .656613 ; 2245.737662$ 15.659547; 23 45.744143 15.681007; 24 45.741272 15.663415; 25 45.741228 15.663497; 2645.739455 15.663512; 2745.744853 15.649172; 28 45.745973 15.642012; $2945.7563915 .624458 ; 3045.768983$ 15.4521663; 31 45.757715 15.56269; 32 45.7640917 15.5798533; 3345.75733 15.373283; 34 45.768115 15.36673; 3545.7645447 15.3672001; 3645.768759 15.3666986; 37 45.7158634 15.4626465; 38 45.7679917 15.4508067; 3945.730737 15.650652; 4045.733065 15.654508; 41 45.744617 15.645718; 42 45.746258 15.640677; 43 45.753217 15.64244; 4445.753423 15.643365; 4545.78123815 .643157$.

Table 8 (Tabela 8): Ass. Cardamino savensi-Fagetum.

\begin{tabular}{lccccccccccccccc}
\hline Relevé No. & 1 & 2 & 3 & 4 & 5 & 6 & 7 & 8 & 9 & 10 & 11 & 12 & 13 & 14 & 15 \\
Date 2010 (Day/Month) & $9 / 6$ & $9 / 6$ & $9 / 6$ & $9 / 6$ & $9 / 6$ & $26 / 5$ & $26 / 5$ & $26 / 5$ & $26 / 5$ & $26 / 5$ & $26 / 5$ & $26 / 5$ & $26 / 5$ & $26 / 5$ & $26 / 5$ \\
Elevation (m) & 538 & 791 & 649 & 540 & 734 & 988 & 1035 & 1025 & 1029 & 1026 & 706 & 695 & 997 & 1044 & 1030 \\
Aspect $\left({ }^{\circ}\right)$ & 360 & 320 & 150 & 45 & 360 & 135 & 315 & 315 & 135 & 90 & 360 & 80 & 70 & 110 & 15 \\
Slope $\left({ }^{\circ}\right)$ & 60 & 40 & 35 & 30 & 35 & 20 & 5 & 5 & 15 & 10 & 25 & 60 & 5 & 5 & 15 \\
Cover of layers (\%): & & & & & & & & & & & & & & & \\
Tree (high) & 100 & 100 & 100 & 100 & 100 & 100 & 90 & 90 & 100 & 90 & 100 & 100 & 80 & 90 & 100 \\
Tree (middle) & 20 & 5 & 5 & 20 & 20 & 20 & 30 & 30 & 20 & 30 & 20 & 10 & 30 & 10 & 10 \\
Shrub & 5 & 5 & 3 & 5 & 5 & 15 & 20 & 10 & 15 & 20 & 10 & 5 & 40 & 10 & 30 \\
Herb & 80 & 70 & 70 & 90 & 80 & 90 & 100 & 100 & 60 & 100 & 60 & 80 & 90 & 90 & 80 \\
\hline
\end{tabular}

Charact. and diff. species of the ass.

Cardamine trifolia

Veronica montana

Cardamine waldsteinii

Lamio orvalae-Fagenion

Cardamine kitaibelii

Lamium orvala

Scopolia carniolica

\begin{tabular}{|c|c|c|c|c|c|c|c|c|c|c|c|c|c|c|}
\hline \multirow[t]{3}{*}{ C } & . & . & . & + & . & + & + & + & . & 1 & . & . & + & + \\
\hline & . & . & . & + & . & . & + & . & . & . & . & . & + & . \\
\hline & . & . & . & + & + & . & . & . & . & . & . & . & . & . \\
\hline \multirow[t]{3}{*}{ C } & . & . & . & 1 & + & + & + & + & + & 2 & + & . & . & + \\
\hline & + & . & + & + & 1 & . & . & . & . & . & - & + & . & . \\
\hline & 2 & . & . & + & . & . & . & . & . & . & . & + & . & . \\
\hline
\end{tabular}

Aremonio-Fagion

Staphylea pinnata

Cyclamen purpurascens

Vicia oroboides

Cardamine enneaphyllos

Isopyrum thalictroides 


\begin{tabular}{cccccccccccccccccccccc}
16 & 17 & 18 & 19 & 20 & 21 & 22 & 23 & 24 & 25 & 26 & 27 & 28 & 29 & 30 & 31 & 32 & 33 & 34 & 35 & 36 & 37 \\
$26 / 5$ & $26 / 5$ & $21 / 5$ & $21 / 5$ & $21 / 5$ & $21 / 5$ & $21 / 5$ & $21 / 5$ & $29 / 6$ & $29 / 6$ & $29 / 6$ & $29 / 6$ & $29 / 6$ & $29 / 6$ & $29 / 6$ & $29 / 6$ & $29 / 6$ & $29 / 6$ & $29 / 6$ & $29 / 6$ & $28 / 5$ & $28 / 5$ \\
1044 & 1045 & 686 & 679 & 659 & 668 & 708 & 685 & 845 & 880 & 882 & 853 & 834 & 836 & 839 & 859 & 879 & 861 & 873 & 832 & 840 & 850 \\
150 & 140 & 30 & 330 & 20 & 70 & 230 & 120 & 6 & 214 & 275 & 309 & 146 & 125 & 190 & 10 & 310 & 300 & 40 & 20 & 360 & 315 \\
20 & 40 & 20 & 30 & 40 & 15 & 35 & 15 & 25 & 15 & 15 & 10 & 30 & 15 & 15 & 50 & 40 & 20 & 5 & 40 & 5 & 25 \\
& & & & & & & & & & & & & & & & & & & & & \\
90 & 100 & 100 & 90 & 100 & 100 & 100 & 100 & 90 & 90 & 100 & 100 & 80 & 80 & 90 & 100 & 100 & 90 & 100 & 90 & & 5 \\
20 & 20 & 10 & 10 & 20 & 5 & 5 & 20 & 20 & 20 & 15 & 20 & 30 & 20 & 2 & 10 & 5 & 15 & 3 & 5 & & 5 \\
10 & 3 & 30 & 10 & 5 & 30 & 10 & 50 & 5 & 5 & 5 & 40 & 70 & 60 & 60 & 5 & 2 & 5 & 5 & 10 & & 90 \\
50 & 70 & 90 & 100 & 50 & 100 & 100 & 70 & 50 & 70 & 80 & 80 & 80 & 80 & 60 & 40 & 60 & 90 & 80 & 90 & \\
\hline
\end{tabular}




\section{$\begin{array}{llllllllllllllll}\text { Relevé No. } & 1 & 2 & 3 & 4 & 5 & 6 & 7 & 8 & 9 & 10 & 11 & 12 & 13 & 14 & 15\end{array}$}

Aremonia agrimonoides

Aposeris foetida

Omphalodes verna

Helleborus niger

Hacquetia epipactis

Euphorbia carniolica

Knautia drymeia

Epimedium alpinum

\section{Fagetalia}

Fagus sylvatica

Fagus sylvatica

Fagus sylvatica

Fagus sylvatica

Fraxinus excelsior

Fraxinus excelsior

$\begin{array}{llllllllllllllll}\text { a1 } & 4 & 5 & 5 & 3 & 4 & 5 & 5 & 5 & 5 & 5 & 5 & 5 & 5 & 4 & 5 \\ \mathrm{a} 2 & 2 & 1 & 1 & 2 & 1 & 2 & 2 & 2 & 2 & 2 & 2 & 1 & 2 & 1 & 1 \\ \mathrm{~b} & + & + & + & + & + & 1 & 1 & + & 1 & 2 & 1 & + & 2 & 1 & 2 \\ \mathrm{c} & . & . & . & . & + & . & . & . & . & . & + & . & + & . & +\end{array}$

Lonicera alpigena

Daphne mezereum

Galium odoratum

Cardamine bulbifera

Dryopteris filix-mas

Paris quadrifolia

Athyrium filix-femina

Polygonatum multiflorum

Carex sylvatica

Mycelis muralis

Mercurialis perennis

Actaea spicata

Lamium galeobdolon

Prenanthes purpurea

Lilium martagon

Ranunculus lanuginosus

Circaea lutetiana

Euphorbia amygdaloides

Pulmonaria officinalis

Brachypodium sylvaticum

Leucojum vernum

Scrophularia nodosa

Sanicula europaea

Allium ursinum

Epilobium montanum

Euphorbia dulcis

Viola reichenbachiana

Epipactis helleborine

Corydalis bulbosa

Hordelymus europaeus

Ranunculus ficaria

Neottia nidus-avis

Asarum europaeum

Festuca altissima 


\section{Relevé No. $\begin{array}{lllllllllllllll}1 & 2 & 3 & 4 & 5 & 6 & 7 & 8 & 9 & 10 & 11 & 12 & 13 & 14 & 15\end{array}$ \\ Carpino-Fagetea}

Acer pseudoplatanus

Acer pseudoplatanus

Acer pseudoplatanus

Acer pseudoplatanus

Acer platanoides

Acer platanoides

Acer platanoides

Ulmus glabra

Ulmus glabra

Ulmus glabra

Abies alba

Abies alba

Abies alba

Carpinus betulus

Carpinus betulus

Lonicera xylosteum

Euonymus latifolius

Rosa arvensis

Prunus avium

Acer campestre

Sorbus aria

Euonymus verrucosus

Anemone nemorosa

Arum maculatum

Hedera helix

Polystichum aculeatum

Symphytum tuberosum

Glechoma hirsuta

Stellaria nemorum

Luzula luzuloides

Polystichum setiferum

Cephalanthera damasonium

Polygonatum verticillatum

Aruncus dioicus

Dryopteris dilatata

Galium sylvaticum

Milium effusum

Lunaria rediviva

Hepatica nobilis

Melica uniflora

Adoxa moschatellina

Aconitum lycoctonum

Carex digitata

Phegopteris connectilis

Luzula sylvatica

Stachys sylvatica

\section{Vaccinio-Piceetea}

Picea abies

Picea abies

$$
\begin{aligned}
& \begin{array}{llllllllllllllll}
\mathrm{a} 1 & 1 & \cdot & \cdot & 3 & 1 & \cdot & + & 1 & \cdot & \cdot & \cdot & \cdot & \cdot & 2 & . \\
\mathrm{a} 2 & + & \cdot & \cdot & \cdot & + & \cdot & + & \cdot & \cdot & + & \cdot & + & 2 & + & . \\
\mathrm{b} & \cdot & + & \cdot & \cdot & + & + & \cdot & + & + & + & + & + & 2 & + & 1
\end{array} \\
& \cdot \cdot \cdot \cdot \\
& \text { a2 } \\
& \text { b } \\
& \text { a1 } \\
& \text { a2 } \\
& \text { b } \\
& \text { a1 } \\
& \text { a2 } \\
& \text { b } \\
& \text { a2 } \\
& \text { b } \\
& \text { c } \\
& 2 .+2 \\
& \text {. }+.+ \\
& 2
\end{aligned}
$$

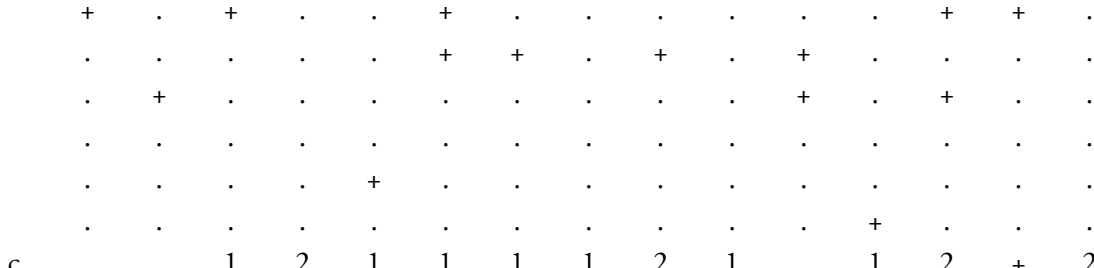

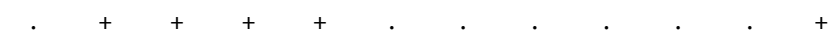

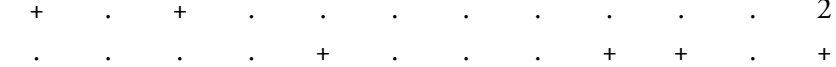

$$
\begin{aligned}
& + \\
& 1
\end{aligned}
$$




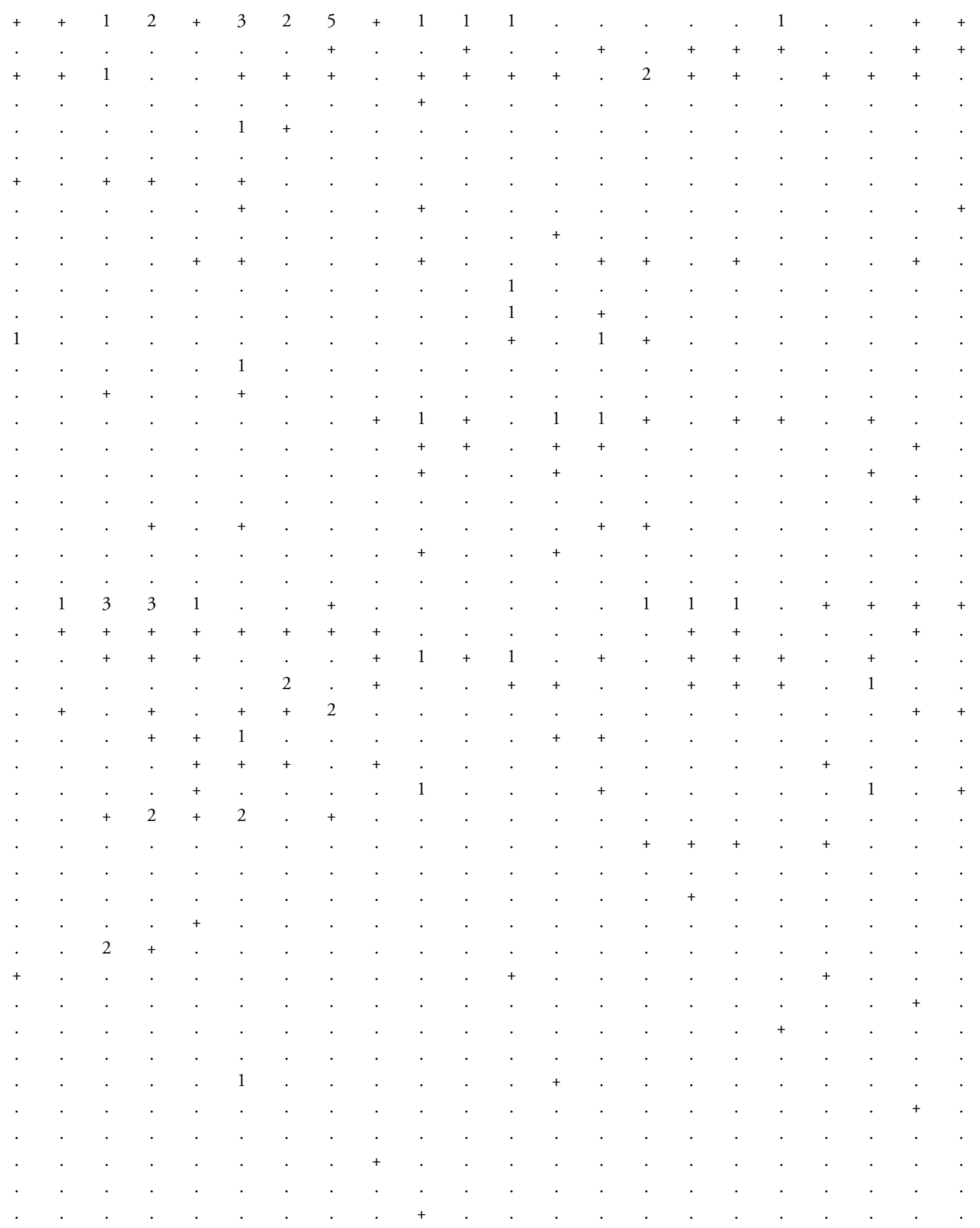




\section{Relevé No.}

$\begin{array}{lllllllllllllll}1 & 2 & 3 & 4 & 5 & 6 & 7 & 8 & 9 & 10 & 11 & 12 & 13 & 14 & 15\end{array}$

Oxalis acetosella

Quercetea pubescentis

Fraxinus ornus

c

Cornus mas

Acer obtusatum

Tamus communis

Festuca heterophylla

Mercurialis ovata

Erico-Pinetea

Genista januensis

Carex alba

Epilobietea angustifolii

Senecio ovatus

Heracleum sphondylium

Geranium robertianum

Urtica dioica

Myosotis sylvatica

Impatiens noli-tangere

Geranium phaeum

Alliaria petiolata

Salvia glutinosa

Silene dioica

Anthriscus sylvestris

Petasites albus

Geum urbanum

Lamium maculatum

Atropa bella-donna

Aegopodium podagraria

Glechoma hederacea

Hypericum hirsutum

Eupatorium cannabinum

Fragaria vesca

\section{Rhamno-Prunetea}

Sorbus aucuparia

Sorbus aucuparia

b

Rubus hirtus

Corylus avellana

Solidago virgaurea

Robinietea

Sambucus nigra

Clematis vitalba

Sambucus racemosa

Rubus idaeus

Trifolio-Geranietea

Potentilla micrantha

Laser trilobum

Veronica chamaedrys

Melampyrum pratense

Tanacetum corymbosum

$$
\begin{aligned}
& \mathrm{a} 2 \\
& \mathrm{~b}
\end{aligned}
$$$$
\text { b }
$$$$
\text { c }
$$ 


\begin{tabular}{|c|c|c|c|c|c|c|c|c|c|c|c|c|c|c|c|c|}
\hline Relevé No. & & 1 & 2 & 3 & 4 & 5 & 6 & 7 & 8 & 9 & 10 & 11 & 12 & 13 & 14 & 15 \\
\hline \multicolumn{17}{|l|}{ Mulgedio-Aconitetea } \\
\hline Phyteuma ovatum & c & . & . & . & . & + & & + & . & . & . & . & + & + & . & . \\
\hline Doronicum austriacum & & . & . & . & . & . & & . & + & . & + & + & . & . & . & . \\
\hline Saxifraga rotundifolia & & . & . & . & . & . & & . & . & . & . & . & + & . & . & . \\
\hline Thalictrum aquilegiifolium & & . & . & . & . & . & & . & & . & . & . & . & . & + & . \\
\hline Veratrum album & & . & . & . & . & . & . & . & . & . & . & . & . & + & . & . \\
\hline \multicolumn{17}{|l|}{ Festuco-Brometea } \\
\hline Veronica austriaca & c & . & . & . & . & . & . & . & . & . & . & . & + & . & . & . \\
\hline Dorycnium germanicum & & . & . & . & . & . & & . & . & . & . & . & . & . & . & . \\
\hline Carex flacca & & . & . & . & . & . & & . & . & . & . & . & . & . & . & . \\
\hline Listera ovata & & . & . & . & . & . & & . & . & . & . & . & . & . & . & . \\
\hline \multicolumn{17}{|l|}{ Molinio-Arrhenatheretea } \\
\hline Platanthera bifolia & c & . & + & . & . & . & & . & & . & & + & . & . & . & . \\
\hline Gentiana asclepiadea & & + & . & . & . & + & & . & . & . & . & + & + & . & . & . \\
\hline \multicolumn{17}{|l|}{ Asplenietea trichomanis } \\
\hline Asplenium scolopendrium & c & 1 & . & + & + & + & & . & . & . & . & . & . & . & . & . \\
\hline Polypodium vulgare & & . & . & . & . & . & & . & ${ }^{2}$ & + & . & . & . & . & . & . \\
\hline Asplenium trichomanes & & . & . & . & . & . & & . & 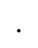 & . & . & . & . & . & . & . \\
\hline \multicolumn{17}{|l|}{ Other species } \\
\hline Polystichum lonchitis & c & . & . & . & . & . & & . & & . & . & . & . & . & . & . \\
\hline Chrysosplenium alternifolium & c & . & . & . & + & . & & . & . & . & . & . & . & . & . & . \\
\hline Pteridium aquilinum & c & . & . & . & . & . & & . & & . & . & . & . & . & . & . \\
\hline Veronica officinalis & c & . & . & . & . & . & & . & & . & . & . & . & . & . & . \\
\hline Rubus sp. & c & . & . & . & . & . & & . & & . & . & . & . & . & . & . \\
\hline Solanum dulcamara & c & . & . & . & . & . & & . & & . & . & . & . & . & . & . \\
\hline Festuca sp. & c & . & . & . & . & . & & . & & . & & . & . & 1 & . & \\
\hline
\end{tabular}

Legend: a1 - High tree layer, a2 - Middle tree layer, b - Shrub layer, c - Herb layer.

Coordinates of the relevés:

1 45.756343 15.5842277; 2 45.7461051 15.6005391; 3 45.7655307 15.5873577; 4 45.7555467 15.58626;

$\mathbf{5} 45.7466483$ 15.5993583; 6 45.758022 15.335857; 7 45.758885 15.332405; 8 45.754593 15.326137;

$945.75460715 .32661 ; 1045.75355515 .319015 ; 1145.725893$ 15.36985; 1245.7608788 15.3679925;

13 45.7585968 15.3355357; 14 45.7594223 15.3320524; 15 45.7546332 15.3269713; 1645.7536615 15.3274339;

17 45.7543532 15.3194783; 1852145.8201543 15.5186086; 1952145.8197857 15.5191784;

2052145.8192367 15.5206333; 2152145.8195734 15.5201301; 22521 45.8168468 15.5139762;

2352145.8164123 15.5142639; 2445.7907867 15.40765; 25 45.7876567 15.411285; 2645.79328 15.4151583;

2745.796175 15.415975; $2845.775186715 .4383017 ; 2945.7753617$ 15.4383383; 3045.7646681 15.3828233;

31 45.7909205 15.4047275; 32 45.7879779 15.4109376; 33 45.7939337 15.4145938; 34 45.795331 15.4174931;

3545.7753861 15.4408889; 3645.7495459 15.6098554; 3745.749680615 .608772$. 


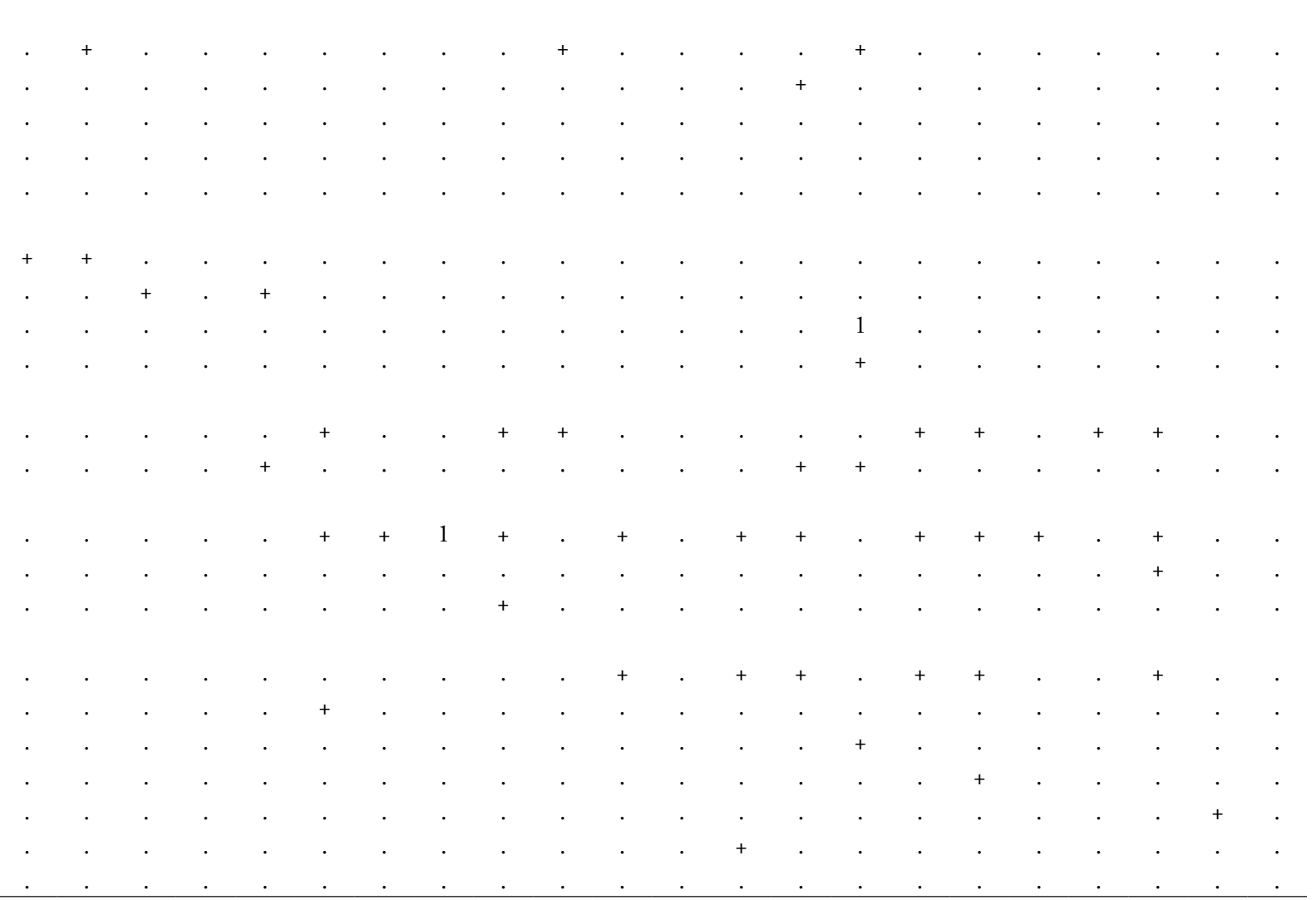

